\title{
Convergence analysis of high-order time-splitting generalized-Laguerre-Fourier-Hermite pseudo-spectral methods for rotational Gross-Pitaevskii equations
}

\author{
Harald Hofstätter • Othmar Koch • \\ Mechthild Thalhammer
}

\begin{abstract}
A convergence analysis of time-splitting pseudo-spectral methods adapted for time-dependent Gross-Pitaevskii equations with additional rotation term is given. For the time integration high-order exponential operator splitting methods are studied, and the space discretization relies on the generalized-Laguerre-Fourier spectral method with respect to the $(x, y)$ variables as well as the Hermite spectral method in the $z$-direction. Essential ingredients in the stability and error analysis are a general functional analytic framework of abstract nonlinear evolution equations, fractional power spaces defined by the principal linear part, a Sobolev-type inequality in a curved rectangle, and results on the asymptotical distribution of the nodes and weights associated with Gauß-Laguerre quadrature. The obtained global error estimate ensures that the nonstiff convergence order of the time integrator and the spectral accuracy of the spatial discretization are retained, provided that the problem data satisfy suitable regularity requirements. A numerical example confirms the theoretical convergence estimate.
\end{abstract}

We acknowledge financial support by the Austrian Science Fund (FWF) under the projects P21620-N13 and P24157-N13. The final publication is available at Springer via http://dx.doi.org/10.1007/s00211-013-0586-9

Harald Hofstätter (Corresponding author)

Institute for Analysis and Scientific Computing, Vienna University of Technology

Wiedner Hauptstrasse 8-10, A-1040 Wien, Austria

E-mail: hofi@harald-hofstaetter.at

Othmar Koch

Institute for Analysis and Scientific Computing, Vienna University of Technology

Wiedner Hauptstrasse 8-10, A-1040 Wien, Austria

E-mail: othmar@othmar-koch.org

Mechthild Thalhammer

Institut für Mathematik, Leopold-Franzens Universität Innsbruck

Technikerstraße 13/VII, A-6020 Innsbruck, Austria

E-mail: mechthild.thalhammer@uibk.ac.at 
Keywords Time-dependent Gross-Pitaevskii equation with rotation term; Nonlinear Schrödinger equation; Full discretization; Generalized-LaguerreFourier-Hermite pseudo-spectral method; High-order time-splitting method; Stability; Local error; Convergence

Mathematics Subject Classification (2000) 65L05, 65M12, 65J15

\section{Introduction}

Scope of applications. The realization of dilute gaseous Bose-Einstein condensation in physical experiments has received great attention among physicists to date. Current research activities aim for a better understanding of the creation and evolution of quantized vortices in rotating Bose-Einstein condensates. The extensive experimental work is supplemented by mathematical investigations, see for instance $[3,4,8,9]$ and references therein.

Time-dependent Gross-Pitaevskii equation with rotation term. At temperatures significantly below the critical temperature of the condensate, certain aspects of the time evolution of a rotating condensate are mathematically described by a nonlinear time-dependent Schrödinger equation for the macroscopic wave function $\psi: \mathbb{R}^{3} \times[0, T] \rightarrow \mathbb{C}:(\xi, t)=(x, y, z, t) \mapsto \psi(\xi, t)$, the time-dependent Gross-Pitaevskii equation with additional rotation term

$$
\mathrm{i} \partial_{t} \psi(\xi, t)=\left(-\frac{1}{2} \Delta+V_{\text {ext }}(\xi)-\Omega L_{z}+\beta|\psi(\xi, t)|^{2}\right) \psi(\xi, t),
$$

subject to asymptotic boundary conditions on the unbounded domain and an initial condition. Here, $V_{\text {ext }}: \mathbb{R}^{3} \rightarrow \mathbb{R}$ denotes an external real-valued potential, which we assume to comprise a scaled harmonic potential that is symmetric with respect to the $(x, y)$-components and an additional sufficiently regular potential $V: \mathbb{R}^{3} \rightarrow \mathbb{R}$

$$
V_{\mathrm{ext}}(\xi)=\frac{1}{2} \gamma\left(x^{2}+y^{2}\right)+\frac{1}{2} \gamma_{z} z^{2}+V(\xi), \quad \gamma, \gamma_{z}>0
$$

The rotation term involves the angular momentum rotation speed $\Omega \in \mathbb{R}$ and the angular momentum operator

$$
L_{z}=-\mathrm{i}\left(x \partial_{y}-y \partial_{x}\right)
$$

Besides, we denote by $\beta \in \mathbb{R}$ the interaction constant arising in the cubic nonlinearity. A discussion of the physical background of this model and a numerical investigation of its solution behavior is found for instance in [4], see also references given therein. 
Abstract evolution equation. For our purpose it is useful to employ a compact reformulation of (1) as nonlinear evolution equation of the form

$$
\mathrm{i} \frac{\mathrm{d}}{\mathrm{d} t} u(t)=A u(t)+B[u(t)] u(t)
$$

for an abstract function $u:[0, T] \rightarrow L^{2}\left(\mathbb{R}^{3}\right): t \mapsto u(t)=\psi(\cdot, t)$. The linear differential operator $A$ is given by

$$
(A u)(\xi)=\left(-\frac{1}{2} \Delta+\frac{1}{2} \gamma\left(x^{2}+y^{2}\right)+\frac{1}{2} \gamma_{z} z^{2}-\Omega L_{z}\right) u(\xi)
$$

and $B$ denotes a nonlinear multiplication operator acting as

$$
(B[u] v)(\xi)=\left(V(\xi)+\beta|u(\xi)|^{2}\right) v(\xi) .
$$

We meanwhile require the domains of $A$ and $B$ to be suitably chosen subspaces of the underlying Hilbert space $\left(L^{2}\left(\mathbb{R}^{3}\right),(\cdot \mid \cdot)_{L^{2}},\|\cdot\|_{L^{2}}\right)$ and give more details below.

Two-dimensional Gross-Pitaevskii equation and reformulation in polar coordinates. In order to reduce technicalities, we find it beneficial to study at first a simplified problem for a function depending on two space variables $\psi: \mathbb{R}^{2} \times[0, T] \rightarrow \mathbb{C}:(\xi, t)=(x, y, t) \mapsto \psi(\xi, t)$. We refer to this problem obtained from (1)-(2) when replacing (1b) and thus (2b) by

$$
\begin{gathered}
V_{\mathrm{ext}}(\xi)=\frac{1}{2} \gamma\left(x^{2}+y^{2}\right)+V(\xi), \\
(A u)(\xi)=\left(-\frac{1}{2} \Delta+\frac{1}{2} \gamma\left(x^{2}+y^{2}\right)-\Omega L_{z}\right) u(\xi),
\end{gathered}
$$

as time-dependent two-dimensional Gross-Pitaevskii equation with rotation term. By introducing polar coordinates

$$
(r, \vartheta) \in[0, \infty) \times[0,2 \pi), \quad(x, y)=(r \cos \vartheta, r \sin \vartheta) \in \mathbb{R}^{2},
$$

the Laplacian in two space dimensions and the angular momentum operator may be rewritten as $\Delta=\partial_{r}^{2}+\frac{1}{r} \partial_{r}+\frac{1}{r^{2}} \partial_{\vartheta}^{2}$ and $L_{z}=-\mathrm{i} \partial_{\vartheta}$, which implies

$$
\begin{aligned}
A & =-\frac{1}{2} \Delta+\frac{1}{2} \gamma\left(x^{2}+y^{2}\right)-\Omega L_{z} \\
& =-\frac{1}{2}\left(\partial_{r}^{2}+\frac{1}{r} \partial_{r}+\frac{1}{r^{2}} \partial_{\vartheta}^{2}-\gamma r^{2}\right)+\mathrm{i} \Omega \partial_{\vartheta} .
\end{aligned}
$$

The construction of a family of eigenfunctions that forms a complete orthonormal system of the Lebesgue space $L^{2}\left(\mathbb{R}^{2}\right)$ is based upon this reformulation, see for instance [24]. 
Time and space discretization. The complexity of the time-dependent threedimensional Gross-Pitaevskii equation with additional rotation term renders its numerical solution a demanding task and requires the use of appropriate space and time discretization methods. For the considered model problem (1) and related time-dependent nonlinear Schrödinger equations a variety of contributions provide numerical comparisons which confirm the favourable behavior of full discretization methods based on higher-order time-splitting and pseudo-spectral methods in accuracy, efficiency, and the conservation of physically relevant quantities; to mention a few we refer to the works $[4,5,7]$. Numerical evidence is complemented by a rigorous analysis of the convergence behavior of time-splitting pseudo-spectral methods for certain classes of timedependent nonlinear Schrödinger equations. In the seminal work [17] the stability and error behavior of the second-order Strang splitting method for the time integration of the cubic Schrödinger equation is analyzed, and in [10] a convergence estimate for full discretizations of the time-dependent Gross-Pitaevskii equation (without rotation term) based on the Strang splitting method and the Hermite pseudo-spectral method is deduced. The recent contribution [14] extends the approach to high-order splitting methods for the time integration of the MCTDHF equations, and in [23] a stability and error analysis of high-order time-splitting methods combined with pseudo-spectral methods (Fourier, Sine, Hermite) is given for the time-dependent Gross-Pitaevskii equation (without rotation term).

High-order time-splitting generalized-Laguerre-Fourier-Hermite pseudospectral methods. In the present work, we consider time-splitting pseudospectral methods that are well adapted for the numerical solution of the time-dependent three-dimensional Gross-Pitaevskii equations involving an additional rotation term. More precisely, high-order exponential operator splitting methods with variable time stepsizes are applied for the time integration, and the space discretization of (1)-(2) relies on a combination of the generalized-Laguerre-Fourier spectral method with respect to the $(x, y)$ variables and the Hermite spectral method with respect to the $z$-direction, see for instance $[4,7]$.

Basic tools. In the following, we review basic tools that are needed in the specification of high-order time-splitting generalized-Laguerre-Fourier-Hermite pseudo-spectral methods for the time-dependent three-dimensional GrossPitaevskii equation with rotation term; detailed explanations are included in subsequent sections.

Exponential operator splitting methods for the time integration of nonlinear evolution equations (2) utilize the natural decomposition of the operator defining the right-hand side of the differential equation into two parts and rely on the presumption that the associated subproblems

$$
\mathrm{i} \frac{\mathrm{d}}{\mathrm{d} t} v(t)=A v(t), \quad \mathrm{i} \frac{\mathrm{d}}{\mathrm{d} t} w(t)=B[w(t)] w(t),
$$


can be resolved numerically in an efficient manner. In particular, in the context of the time-dependent Gross-Pitaevskii equation with rotation term (1), we may choose the operators $A$ and $B$ in such a way that the exact solution of the subproblem involving the dominant linear part $(2 \mathrm{~b})$ is obtained by a spectral representation of the prescribed initial value. Due to a particular invariance property, the subproblem involving the remaining terms (2c) reduces to a linear differential equation whose exact solution is given by pointwise multiplications.

(i) Linear subproblem. For the resolution of the linear subproblem we make use of the fact that $A$ extends to a densely defined self-adjoint operator $A: D(A) \subset L^{2}\left(\mathbb{R}^{3}\right) \rightarrow L^{2}\left(\mathbb{R}^{3}\right)$ and that there exists a family of associated eigenfunctions $\left(\mathcal{B}_{\mu}\right)_{\mu \in \mathcal{M}}$ over the index set $\mathcal{M}=\mathbb{N} \times \mathbb{Z} \times \mathbb{N}$ which forms a complete orthonormal system of the underlying Hilbert space $L^{2}\left(\mathbb{R}^{3}\right)$, see also (2b). These eigenfunctions $\mathcal{B}_{\mu}: \mathbb{R}^{3} \rightarrow \mathbb{C}$ are given by the generalizedLaguerre polynomials and the Hermite polynomials $\left(\xi=(x, y, z) \in \mathbb{R}^{3}\right.$, $\mu=(k, m, \ell) \in \mathcal{M})$

$$
\begin{gathered}
\mathcal{B}_{\mu}(\xi)=\mathcal{L}_{k m}^{\gamma}(x, y) \mathcal{H}_{\ell}^{\gamma_{z}}(z) \\
\mathcal{L}_{k m}^{\gamma}(r \cos \vartheta, r \sin \vartheta)=\frac{1}{\sqrt{\pi C_{k}^{|m|}}} \gamma^{\frac{1}{2}(|m|+1)} r^{|m|} \mathrm{e}^{\mathrm{i} m \vartheta-\frac{1}{2} \gamma r^{2}} L_{k}^{|m|}\left(\gamma r^{2}\right) \\
\mathcal{H}_{\ell}^{\gamma_{z}}(z)=\frac{1}{\sqrt{2^{\ell} \ell}} \sqrt[4]{\frac{\pi}{\gamma_{z}}} \mathrm{e}^{-\frac{1}{2} \gamma_{z} z^{2}} H_{\ell}\left(\sqrt{\gamma_{z}} z\right) .
\end{gathered}
$$

We point out that the complex-valued functions $\mathcal{L}_{k m}^{\gamma}: \mathbb{R}^{2} \rightarrow \mathbb{C}$ are defined in terms of polar coordinates which leads to a coupling in the $(x, y)$-coordinates and increases the complexity of the analysis compared to other spectral methods such as the Fourier, Sine, or Hermite spectral method. In order to ensure that the corresponding eigenvalues $\left(\lambda_{\mu}\right)_{\mu \in \mathcal{M}}$ satisfying $(\mu=(k, m, \ell) \in \mathcal{M})$

$$
A \mathcal{B}_{\mu}=\lambda_{\mu} \mathcal{B}_{\mu}, \quad \lambda_{\mu}=(2 k+|m|+1) \gamma-m \Omega+\left(\ell+\frac{1}{2}\right) \gamma_{z},
$$

are positive, we henceforth require the slightly stronger condition

$$
|\Omega|<\gamma
$$

to be satisfied. Via a spectral decomposition of the prescribed initial value $v(0)=v_{0}$, the solution representation

$$
v(t)=\mathrm{e}^{-\mathrm{i} t A} v_{0}=\sum_{\mu \in \mathcal{M}} c_{\mu}\left(v_{0}\right) \mathrm{e}^{-\mathrm{i} t \lambda_{\mu}} \mathcal{B}_{\mu}, \quad c_{\mu}\left(v_{0}\right)=\left(v_{0} \mid \mathcal{B}_{\mu}\right)_{L^{2}},
$$

results. For the numerical realisation, described by the operator $\mathcal{Q}_{M}$

$$
\mathrm{e}^{-\mathrm{i} t A} \mathcal{Q}_{M} v_{0} \approx v(t)=\mathrm{e}^{-\mathrm{i} t A} v_{0},
$$

the infinite index set is restricted to a finite set $\mathcal{M}_{M} \subset \mathcal{M}$, characterised by a positive integer $M \in \mathbb{N}$, and approximations to the spectral coefficients are computed by means of an iterated quadrature formula

$$
\widetilde{c}_{\mu}\left(v_{0}\right)=\sum_{\kappa \in \mathcal{K}_{M}} w_{\kappa} v_{0}\left(\xi_{\kappa}\right) \overline{\mathcal{B}_{\mu}\left(\xi_{\kappa}\right)} \approx c_{\mu}\left(v_{0}\right)=\int_{\mathbb{R}^{3}} v_{0}(\xi) \overline{\mathcal{B}_{\mu}(\xi)} \mathrm{d} \xi
$$


with weights and nodes $\left(w_{\kappa}, \xi_{\kappa}\right)_{\kappa \in \mathcal{K}_{M}}$ related to Gauß-Laguerre quadrature, the trapezoidal rule, and Gauß-Hermite quadrature.

(ii) Nonlinear subproblem. Making use of the fact that the remainder involving part of the external potential as well as the cubic nonlinearity satisfies the special invariance property $\frac{\mathrm{d}}{\mathrm{d} t} B[w(t)]=0$, the nonlinear subproblem reduces to a linear evolution equation with exact solution given by pointwise multiplication

$$
w(t)=\mathrm{e}^{-\mathrm{i} t B[w(0)]} w(0),
$$

see also (2c).

Fully discrete approximations $\left(u_{M}^{n}\right)_{n=1}^{N}$ to the exact solution values $\left(u\left(t_{n}\right)\right)_{n=1}^{N}$ at certain time grid points $0=t_{0}<\cdots<t_{n}<\cdots<t_{N}=T$ with corresponding time increments $\tau_{n}=t_{n+1}-t_{n}$ rely on suitable compositions of the numerical solutions to the subproblems. Starting from a given suitably chosen initial approximation, the new iterates are computed by a recurrence of the form

$$
u_{M}^{n+1}=\mathcal{F}_{M}\left(\tau_{n}, u_{M}^{n}\right) .
$$

For instance, an application of the widely used Strang splitting method combined with the generalized-Laguerre-Fourier-Hermite pseudo-spectral method for the space discretization leads to the relation

$$
\begin{gathered}
u_{M}^{n+1}=\mathcal{F}_{M}\left(\tau_{n}, u_{M}^{n}\right)=\mathrm{e}^{-\frac{1}{2} \mathrm{i} \tau_{n} A} \mathcal{Q}_{M} \mathrm{e}^{-\mathrm{i} \tau_{n} B\left[U_{M}^{n}\right]} U_{M}^{n}, \\
U_{M}^{n}=\mathrm{e}^{-\frac{1}{2} \mathrm{i} \tau_{n} A} \mathcal{Q}_{M} u_{M}^{n} .
\end{gathered}
$$

In order to deduce a global error estimate for the fully discrete solution, it is advantageous to introduce the corresponding time-discrete solution $\left(u^{n}\right)_{n=0}^{N}$ given by

$$
u^{n+1}=\mathcal{S}\left(\tau_{n}, u^{n}\right)=\mathrm{e}^{-\frac{1}{2} \mathrm{i} \tau_{n} A} \mathrm{e}^{-\mathrm{i} \tau_{n} B\left[U^{n}\right]} U^{n}, \quad U^{n}=\mathrm{e}^{-\frac{1}{2} \mathrm{i} \tau_{n} A} u^{n},
$$

and to study first the contribution of the time discretisation.

Convergence analysis. Our main objective is to provide a convergence analysis for high-order time-splitting generalized-Laguerre-Fourier-Hermite pseudospectral methods applied to the time-dependent three-dimensional GrossPitaevskii equation with rotation term, in order to justify the use of this class of numerical methods for practical applications. In particular, we identify regularity requirements on the data of the problem, inherited by the exact solution, which ensure that the nonstiff order of convergence of the time integrator is retained; for less regular solutions an order reduction and thus a loss of accuracy may be encountered, see $[22,23]$ for numerical illustrations in the context of time-dependent linear Schrödinger equations and Gross-Pitaevskii equations (without rotation term). We extend the approach in $[10,14,17]$ and in particular employ techniques that are closely related to the previous work [23], where high-order time-splitting methods and different pseudo-spectral methods (Fourier, Sine, Hermite) are analyzed for time-dependent Gross-Pitaevskii 
equations (without rotation term). However, the complexity of the generalizedLaguerre-Fourier-Hermite pseudo-spectral space discretization implies a considerable increase of technicalities. For this reason, we focus on the case of two space dimensions as this constitutes the main challenge and indicate the extension to three space dimensions based on Hermite basis functions for the $z$ variable. Moreover, as a treatment of high-order time-splitting methods would require to introduce the formal calculus of Lie-derivaties, we include a detailed analysis for the Strang splitting method; the generalization to high-order splitting methods is then in the lines of $[14,23]$.

Outline. The present manuscript has the following structure. In Section 2 we state the main result, a global error estimate for high-order time-splitting generalized-Laguerre-Fourier-Hermite pseudo-spectral methods applied to the time-dependent Gross-Pitaevskii equations involving an additional rotation term and its analogue for the two-dimensional case. In Section 3 we illustrate the theoretical convergence estimate by a numerical example. In Section 4, we collect prerequisites related to the spatial discretization by the generalized-Laguerre-Fourier pseudo-spectral method; following [23] we employ a general functional analytic framework that exposes the similarities between different pseudo-spectral methods. Section 5 is devoted to the derivation of the convergence result for the Strang splitting generalizedLaguerre-Fourier pseudo-spectral method applied to the two-dimensional time-dependent Gross-Pitaevskii equations with rotation term and the extension to three space dimensions.

Notation. Throughout, we employ standard notations and results for Lebesgue and Sobolev spaces, see also [2]. For notational convenience, we do not distinguish between the spatial variables and the associated multiplication operators; for instance, we write $x f$ for the function $x \mapsto x f(x, y)$. Besides, we denote by $\mathbb{N}=\{n \in \mathbb{Z}: n \geq 0\}$ the set of non-negative integer numbers.

\section{Convergence result}

In this section, we state our main result, a global error estimate for high-order time-splitting generalized-Laguerre-Fourier-Hermite pseudo-spectral methods applied to the time-dependent Gross-Pitaevskii equation involving an additional rotation term, see (1)-(2).

Preliminary remarks. We recall the abbreviation $\left(u_{M}^{n}\right)_{n=0}^{N}$ for the fully discrete solution, exemplified in (5) for the Strang splitting method; its timediscrete analogue with starting value $u^{0}=u_{M}^{0}$ is denoted by $\left(u^{n}\right)_{n=0}^{N}$. The choice of the finite index set $\mathcal{M}_{M}$, which reflects the number of basis functions used, determines the accuracy of the spatial discretization. For ease of notation, we assume that the space discretization parameters corresponding to the $(x, y, z)$-components are roughly proportional such that it suffices to 
indicate the dependence on a single positive integer $M \in \mathbb{N}$; in the context of the time-dependent two-dimensional Gross-Pitaevskii equation the space discretization parameter corresponding to the $z$-direction does not occur. The fractional power spaces associated with the linear differential operator $(2 \mathrm{~b})$ are introduced in Section 4.2; in particular, the space $X_{0}$ coincides with the underlying Lebesgue space in three or two space dimensions, respectively. For notational simplicity, despite the fact that the numerical evolution operators are nonlinear in the second argument we henceforth write

$$
\mathcal{F}_{M}(\tau) u=\mathcal{F}_{M}(\tau, u), \quad \mathcal{S}(\tau) u=\mathcal{S}(\tau, u),
$$

for short.

Global error estimate. A standard idea in the derivation of a global error bound for the fully discrete solution is to interpose the values of the corresponding time-discrete solution to obtain the estimate

$$
\left\|u_{M}^{n}-u\left(t_{n}\right)\right\|_{X_{0}} \leq\left\|u_{M}^{n}-u^{n}\right\|_{X_{0}}+\left\|u^{n}-u\left(t_{n}\right)\right\|_{X_{0}}
$$

by the triangle inequality. This approach permits to base the convergence analysis on the derivation of stability results and local error expansions; rewriting the first difference by means of a telescopic identity leads to (in compact formally linear notation)

$$
u_{M}^{n}-u^{n}=\left(\mathcal{Q}_{M}-I\right) u^{n}+\sum_{j=0}^{n-1} \prod_{\ell=j+1}^{n-1} \mathcal{F}_{M}\left(\tau_{\ell}\right)\left(\mathcal{F}_{M}\left(\tau_{j}\right) u^{j}-\mathcal{Q}_{M} \mathcal{S}\left(\tau_{j}\right) u^{j}\right)
$$

and similarly for the second contribution. For the special case of the secondorder Strang splitting method and two space dimensions the needed auxiliary results are deduced in Section 5.2, and a bound for the contribution of the semi-discretization in time is provided in Section 5.1; the generalization to higher-order time-splitting methods follows the arguments detailed in [23] for space discretizations based on the Fourier, Sine, and Hermite pseudo-spectral methods, respectively, and the extension to the three-dimensional case is indicated in Section 5.3. Altogether, we are able to establish the following convergence result.

Theorem 1 (i) Consider a variable stepsize exponential operator splitting method of nonstiff order $p \in \mathbb{N}_{\geq 1}$ combined with the generalized-LaguerreFourier-Hermite pseudo-spectral method for the full discretization of the timedependent Gross-Pitaevskii equation (1)-(2). Under the assumption that the potential $V$ and the values of the exact solution remain bounded in a fractional power space $X_{q}$ for some $q \geq \max \{2, p\}$, the global error estimate

$\left\|u_{M}^{n}-u\left(t_{n}\right)\right\|_{X_{0}} \leq \mathcal{C}\left(\left\|u_{M}^{0}-u(0)\right\|_{X_{0}}+(\Delta t)^{p}+M^{-r}\right), \quad 0 \leq t_{n} \leq t_{N} \leq T$,

is valid with exponent $r=q-\frac{11}{6}$, where $\Delta t=\max \left\{\tau_{n}: n=0,1, \ldots, N-1\right\}$ denotes the maximal time increment; the arising constant $\mathcal{C}>0$ in particular depends on upper bounds for $\|V\|_{X_{q}}$ and $\max \left\{\|u(t)\|_{X_{q}}: 0 \leq t \leq T\right\}$. 

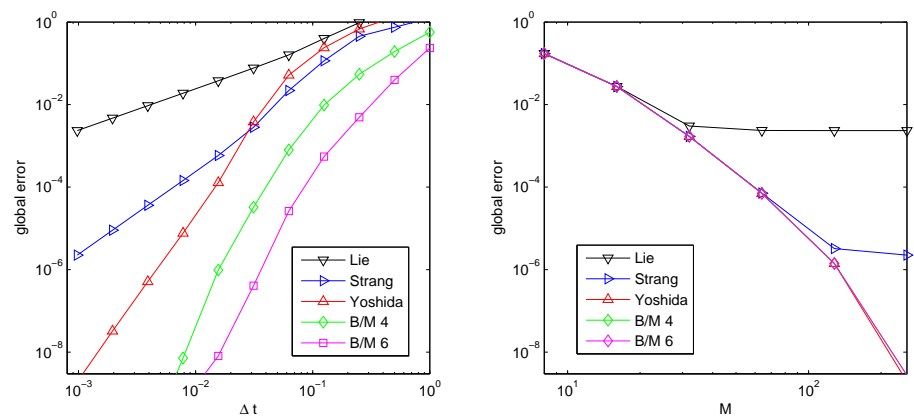

Fig. 1 Full discretisation of the time-dependent two-dimensional Gross-Pitaevskii equation with additional rotation term by the Strang splitting generalized-Laguerre-Fourier pseudospectral method, see (7). Global error versus time stepsize $\Delta t$ (left) and spatial discretization parameter $M$ (right).

(ii) For the generalized-Laguerre-Fourier pseudo-spectral method applied to the time-dependent two-dimensional Gross-Pitaevskii equation the statement holds with exponent $r=q-\frac{9}{6}$.

Remark. The corresponding global error estimate for the Hermite pseudospectral method applied to the time-dependent Gross-Pitaevskii equation without rotation term involves the exponents $r=q-\frac{12}{6}$ in three space dimensions and $r=q-\frac{10}{6}$ in two space dimensions, see [10,23], whereas in both cases the exponent $r=2(q-1)$ is obtained for the Fourier and Sine pseudo-spectral methods, see [23]. We point out that in practical applications the global error is dominated by the temporal error, reflected in the term $\mathcal{C}(\Delta t)^{p}$, and that order reductions $\mathcal{C}(\Delta t)^{k}$ with $k<p$ are observed for less regular solutions. The precise form of the exponent $r$ for different pseudo-spectral methods is primarily of theoretical interest; due to the fact that the contribution $\mathcal{C} M^{-r}$ is comparatively small, even for a low number of basis functions, it seems not feasible to confirm the optimality of the bound in this respect.

\section{Numerical illustration}

The purpose of this section is to confirm the theoretical global error bound of Theorem 1 by a numerical example. For impressive numerical simulations of rotating Bose-Einstein consensates including problems in three space dimensions and coupled systems we refer to [4].

Global error bound. According to [4, Ex.1] we consider the time-dependent Gross-Pitaevskii equation with additional rotation term in two space dimen- 
sions, where we set

$$
\begin{array}{cc}
\gamma=0.8, \quad V(x, y)=0, \quad \Omega=0.5, & \beta=100, \\
V_{\text {ext }}(x, y)=\frac{1}{2} \gamma\left(x^{2}+y^{2}\right)+V(x, y), & (x, y) \in \mathbb{R}^{2}, \\
\psi(x, y, 0)=\frac{1}{\sqrt{\pi}}(x+\mathrm{i} y) \mathrm{e}^{-\frac{1}{2}\left(x^{2}+y^{2}\right)}, & (x, y) \in \mathbb{R}^{2},
\end{array}
$$

see also (1)-(2). For the time integration we apply different splitting methods with constant time stepsize $\Delta t$, the Lie-Trotter splitting (nonstiff order 1), the Strang splitting (nonstiff order 2), the Yoshida splitting (nonstiff order 4), and the methods by Blanes and Moan of orders 4 and 6 , see for instance $[6,13$, 18]. The problem is discretized in space by the generalized-Laguerre-Fourier pseudo-spectral method. In Figure 1 we display the global error at time $t=1$ as a function of the time stepsize for a fixed spatial discretization parameter $M=$ 512 ; furthermore, the global error in dependence of the space discretization parameter $M=2^{m}$ for integers $3 \leq m \leq 8$ is shown, with time stepsize fixed to $\Delta t=2^{-10}=9.765625 \cdot 10^{-4}$. As expected, for the chosen sufficiently regular initial condition the nonstiff temporal orders are retained, and the numerical results confirm the spectral accuracy in space. As expected, the methods by Blanes and Moan are the most accurate, however the high order 6 of the last method cannot be clearly observed in the numerical experiments. We point out that here the global error is dominated by the temporal error; in particular, this behavior is observed for the first-order Lie-Trotter splitting method.

Time evolution. A movie illustrating the evolution of the solution to the timedependent two-dimensional Gross-Pitaevskii equation with additional rotation term is available at http://www.othmar-koch.org/fwf-project2011.html. Following [4, Ex. 1] we choose (7) with modified potential

$$
V(x, y)=\frac{1}{2}\left(\gamma_{y}^{2}-\gamma^{2}\right) y^{2}, \quad(x, y) \in \mathbb{R}^{2}, \quad \gamma_{y}=1.2,
$$

and perform the integration up to time $t=15$. To further illustrate the quality of the approximations, in Figure 2 we compare the solutions and errors for several numerical approximations at $t=15$. In the left column, we plot the densities computed by Lie-Trotter and Strang splitting for different time step-sizes and in the right column we give the errors as compared to a highly accurate reference solution. We observe that the decrease in the step-size indeed reduces the error as predicted and the higher-order method yields more accurate results.

\section{Basic auxiliary results}

In this section, we introduce the employed functional analytic framework and deduce fundamental auxiliary results that are related to the generalizedLaguerre-Fourier pseudo-spectral method for the spatial discretization of the time-dependent two-dimensional Gross-Pitaevskii equation with rotation term. This includes: 


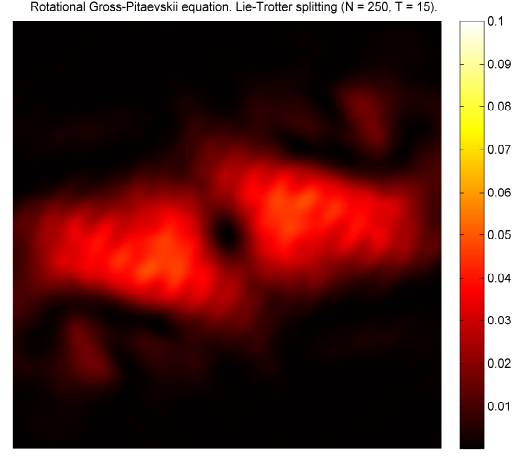

(a) Solution Lie $(N=250)$

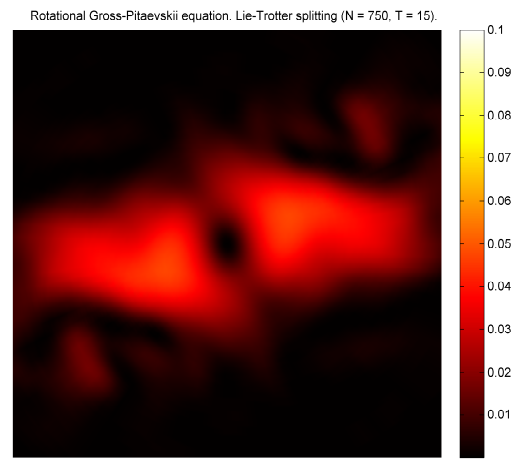

(c) Solution Lie $(N=750)$

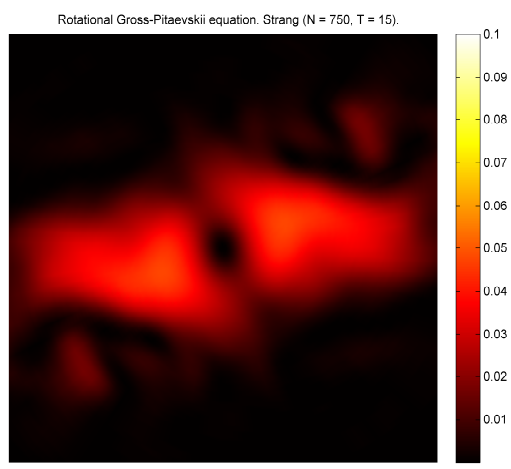

(a) Solution Strang $(N=750)$

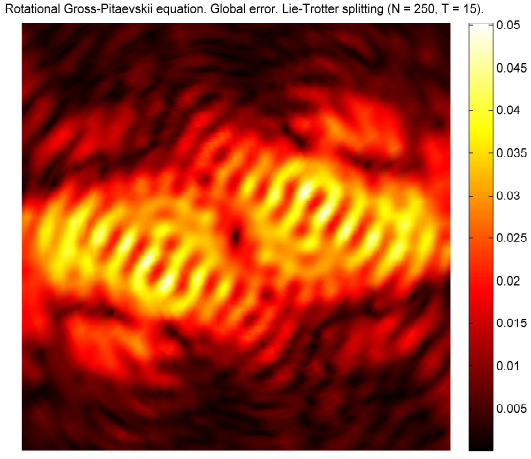

(b) Error Lie $(N=250)$

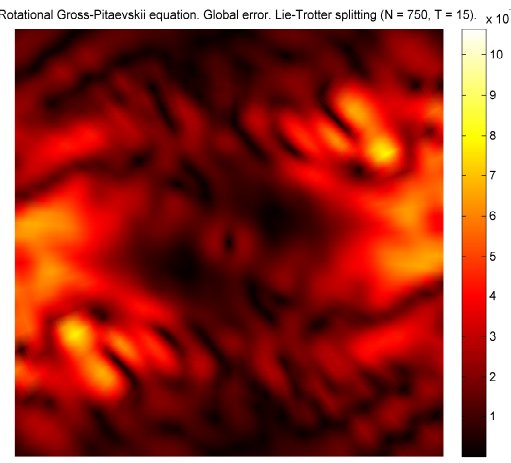

(d) Error Lie $(N=750)$

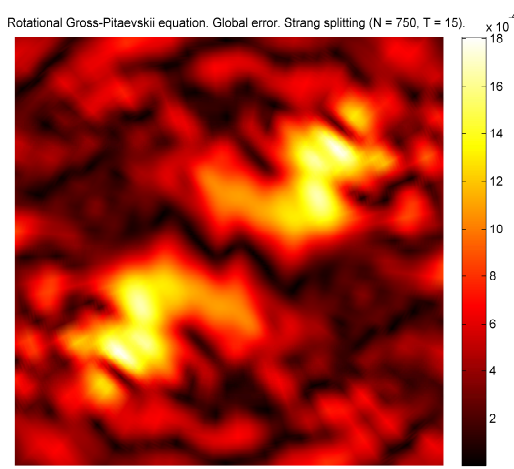

(b) Error Strang $(N=750)$

Fig. 2 Comparison of the approximations at time $t=15$ with $N=250$ or $N=750$ time steps, respectively. Left column: Numerical solution. Right column: Global error as compared to a highly accurate reference solution.

- Results on scaled generalized-Laguerre functions such as relations for partial derivatives of the basis functions (Lemma 1), utilized to relate estimates with 
respect to Sobolev-norms to estimates in fractional power spaces associated with the dominant linear part (Lemma 2). In particular, this implies that fractional power spaces form a normed algebra for positive integer exponents (Lemma 3).

- A Sobolev-type inequality for estimating the maximum value of a function defined on a curved rectangular domain (Lemma 5).

- Results on the asymptotical distribution of the Gauß-Laguerre quadrature nodes and weights, applied to deduce estimates with respect to a related discrete $L^{2}$-norm (Lemma 6).

- A discrete orthogonality relation for the basis functions (Lemma 7) and bounds for the generalized-Laguerre-Fourier spectral interpolant in fractional power spaces (Lemma 8).

Certain technical proofs may be skipped at first reading.

\subsection{Scaled generalized-Laguerre functions}

In the following, we specify the family of eigenfunctions $\left(\mathcal{L}_{k m}^{\gamma}\right)_{(k, m) \in \mathbb{N} \times \mathbb{Z}}$ associated with the differential operator (2f), see also (3). Moreover, we deduce an auxiliary result which allows to express the products $x \mathcal{L}_{k m}^{\gamma}, y \mathcal{L}_{k m}^{\gamma}$ and the partial derivatives $\partial_{x} \mathcal{L}_{k m}^{\gamma}, \partial_{y} \mathcal{L}_{k m}^{\gamma}$ in terms of four basis functions with neighboring indices. For convenience, we first review the employed basic relations for the generalized-Laguerre polynomials and the scaled generalized-Laguerre functions; for further details, see for instance $[1,4,11,19,21,24]$.

Generalized-Laguerre polynomials. The generalized-Laguerre polynomial of degree $k \in \mathbb{N}$, defined by $(k, m \in \mathbb{N}, r \in \mathbb{R})$

$$
L_{k}^{m}(r)=\frac{1}{k !} r^{-m} \mathrm{e}^{r} \frac{\mathrm{d}^{k}}{\mathrm{~d} r^{k}}\left(\mathrm{e}^{-r} r^{k+m}\right),
$$

satisfies the differential equation $(k, m \in \mathbb{N}, r \in \mathbb{R})$

$$
\left(r \frac{\mathrm{d}^{2}}{\mathrm{~d} r^{2}}+(m+1-r) \frac{\mathrm{d}}{\mathrm{d} r}+k\right) L_{k}^{m}(r)=0,
$$

and the orthogonality relation $\left(k, k^{\prime}, m \in \mathbb{N}\right)$

$$
\int_{0}^{\infty} r^{m} \mathrm{e}^{-r} L_{k}^{m}(r) L_{k^{\prime}}^{m}(r) \mathrm{d} r=C_{k}^{m} \delta_{k k^{\prime}}, \quad C_{k}^{m}=\prod_{j=1}^{m}(k+j) .
$$

Furthermore, the relations $(r \in \mathbb{R})$

$$
\begin{aligned}
L_{k}^{m}(r) & =L_{k}^{m+1}(r)-L_{k-1}^{m+1}(r), \quad(k, m) \in \mathbb{N}_{\geq 1} \times \mathbb{N}, \\
\frac{\mathrm{d}}{\mathrm{d} r} L_{k}^{m}(r) & =-L_{k-1}^{m+1}(r), \quad(k, m) \in \mathbb{N}_{\geq 1} \times \mathbb{N}, \\
r L_{k}^{m}(r) & =(k+m) L_{k}^{m-1}(r)-(k+1) L_{k+1}^{m-1}(r), \quad(k, m) \in \mathbb{N} \times \mathbb{N}_{\geq 1}, \\
m L_{k}^{m}(r) & =r L_{k}^{m+1}(r)+(k+1) L_{k+1}^{m-1}(r), \quad(k, m) \in \mathbb{N} \times \mathbb{N}_{\geq 1},
\end{aligned}
$$

are valid. 
Scaled generalized-Laguerre functions. The scaled generalized-Laguerre functions involving a positive weight $\gamma>0$ are defined by $\left(k, m \in \mathbb{N}, r \in \mathbb{R}_{\geq 0}\right)$

$$
\widetilde{L}_{k m}^{\gamma}(r)=\frac{1}{\sqrt{\pi C_{k}^{m}}} \gamma^{\frac{1}{2}(m+1)} r^{m} \mathrm{e}^{-\frac{1}{2} \gamma r^{2}} L_{k}^{m}\left(\gamma r^{2}\right)
$$

the transformation $\varrho=\gamma r^{2}$ leads to the alternative representation

$$
\widetilde{L}_{k m}^{\gamma}(r)=\widetilde{L}_{k m}^{\gamma}\left(\sqrt{\frac{\varrho}{\gamma}}\right)=\sqrt{\frac{\gamma}{\pi C_{k}^{m}}} \varrho^{\frac{m}{2}} \mathrm{e}^{-\frac{1}{2} \varrho} L_{k}^{m}(\varrho) .
$$

The properties of the generalized-Laguerre polynomials imply that the scaled generalized-Laguerre functions obey the differential equation $(k, m \in \mathbb{N}, r \in \mathbb{R})$

$$
\frac{1}{2}\left(-\frac{\mathrm{d}^{2}}{\mathrm{~d} r^{2}}-\frac{1}{r} \frac{\mathrm{d}}{\mathrm{d} r}+\frac{1}{r^{2}} m^{2}+\gamma^{2} r^{2}\right) \widetilde{L}_{k m}^{\gamma}(r)=\gamma(2 k+m+1) \widetilde{L}_{k m}^{\gamma}(r),
$$

and the orthogonality relation $\left(k, k^{\prime}, m \in \mathbb{N}\right)$

$$
2 \pi \int_{0}^{\infty} r \widetilde{L}_{k m}^{\gamma}(r) \widetilde{L}_{k^{\prime} m}^{\gamma}(r) \mathrm{d} r=\delta_{k k^{\prime}},
$$

see also (8).

Related functions. The related complex-valued functions $\mathcal{L}_{k m}^{\gamma}: \mathbb{R}^{2} \rightarrow \mathbb{C}$ are defined in terms of polar coordinates $\left((k, m) \in \mathbb{N} \times \mathbb{Z},(x, y) \in \mathbb{R}^{2}\right)$

$$
\begin{aligned}
\mathcal{L}_{k m}^{\gamma}(x, y) & =\mathcal{L}_{k m}^{\gamma}(r \cos \vartheta, r \sin \vartheta)=\widetilde{L}_{k,|m|}^{\gamma}(r) \mathrm{e}^{\mathrm{i} m \vartheta} \\
& =\widetilde{L}_{k,|m|}^{\gamma}\left(\sqrt{x^{2}+y^{2}}\right)\left(\frac{x+\mathrm{i} y}{\sqrt{x^{2}+y^{2}}}\right)^{m}
\end{aligned}
$$

An application of the corresponding relations for the scaled generalizedLaguerre polynomials implies $\left((k, m) \in \mathbb{N} \times \mathbb{Z},(x, y) \in \mathbb{R}^{2}\right)$

$$
\begin{aligned}
(- & \left.\frac{1}{2} \Delta+\frac{1}{2} \gamma\left(x^{2}+y^{2}\right)-\Omega L_{z}\right) \mathcal{L}_{k m}^{\gamma}(x, y) \\
& =\left(-\frac{1}{2}\left(\partial_{r}^{2}+\frac{1}{r} \partial_{r}+\frac{1}{r^{2}} \partial_{\vartheta}^{2}-\gamma r^{2}\right)+\mathrm{i} \Omega \partial_{\vartheta}\right) \widetilde{L}_{k,|m|}^{\gamma}(r) \mathrm{e}^{\mathrm{i} m \vartheta} \\
& =\left(\frac{1}{2}\left(-\partial_{r}^{2}-\frac{1}{r} \partial_{r}+\frac{1}{r^{2}} m^{2}+\gamma r^{2}\right)-m \Omega\right) \widetilde{L}_{k,|m|}^{\gamma}(r) \mathrm{e}^{\mathrm{i} m \vartheta} \\
& =(\gamma(2 k+|m|+1)-m \Omega) \mathcal{L}_{k m}^{\gamma}(x, y),
\end{aligned}
$$

and yields the orthogonality relations $\left((k, m),\left(k^{\prime}, m^{\prime}\right) \in \mathbb{N} \times \mathbb{Z}\right)$

$$
\begin{aligned}
\left(\mathcal{L}_{k m}^{\gamma} \mid \mathcal{L}_{k^{\prime} m^{\prime}}^{\gamma}\right)_{L^{2}} & =\int_{\mathbb{R}^{2}} \overline{\mathcal{L}_{k m}^{\gamma}(x, y)} \mathcal{L}_{k^{\prime} m^{\prime}}^{\gamma}(x, y) \mathrm{d}(x, y) \\
& =\int_{0}^{\infty} \int_{0}^{2 \pi} r \widetilde{L}_{k,|m|}^{\gamma}(r) \widetilde{L}_{k^{\prime},\left|m^{\prime}\right|}^{\gamma}(r) \mathrm{e}^{\mathrm{i}\left(m^{\prime}-m\right) \vartheta} \mathrm{d} \vartheta \mathrm{d} r \\
& =\delta_{k k^{\prime}} \delta_{m m^{\prime}},
\end{aligned}
$$

see (2f) and (9). 
Pointwise multiplication and partial derivatives. The following auxiliary result is needed in order to establish relations between the norms in Sobolev spaces and fractional power spaces defined by the differential operator (2f). We note that the right-hand sides only differ with respect to constants involving the weight $\gamma>0$ and signs. For convenience we set $\mathcal{L}_{-1, m}^{\gamma}=0$ for $m \in \mathbb{Z}$.

Lemma 1 The following identities are valid for any $(k, m) \in \mathbb{N} \times \mathbb{Z}$

$$
\begin{aligned}
& x \mathcal{L}_{k m}^{\gamma}=\frac{1}{2 \sqrt{\gamma}}\left\{\begin{aligned}
- & \sqrt{k} \mathcal{L}_{k-1, m+1}^{\gamma}+\sqrt{k+m} \mathcal{L}_{k, m-1}^{\gamma} & & \\
& +\sqrt{k+m+1} \mathcal{L}_{k, m+1}^{\gamma}-\sqrt{k+1} \mathcal{L}_{k+1, m-1}^{\gamma}, & & m>0, \\
& \sqrt{k} \mathcal{L}_{k-1,1}^{\gamma}+\sqrt{k+1} \mathcal{L}_{k,-1}^{\gamma} & & \\
& +\sqrt{k+1} \mathcal{L}_{k 1}^{\gamma}-\sqrt{k} \mathcal{L}_{k-1,-1}^{\gamma}, & & m=0, \\
& & & \\
& +\sqrt{k-m-1, m-1}+\sqrt{k-m} \mathcal{L}_{k, m+1}^{\gamma} & & \\
& & &
\end{aligned}\right. \\
& y \mathcal{L}_{k m}^{\gamma}=\frac{\mathrm{i}}{2 \sqrt{\gamma}}\left\{\begin{array}{rlrl} 
& \sqrt{k} \mathcal{L}_{k-1, m+1}^{\gamma}+\sqrt{k+m} \mathcal{L}_{k, m-1}^{\gamma} & \\
& -\sqrt{k+m+1} \mathcal{L}_{k, m+1}^{\gamma}-\sqrt{k+1} \mathcal{L}_{k+1, m-1}^{\gamma}, & & m>0, \\
& \sqrt{k} \mathcal{L}_{k-1,1}^{\gamma}+\sqrt{k+1} \mathcal{L}_{k,-1}^{\gamma} & \\
& -\sqrt{k+1} \mathcal{L}_{k 1}^{\gamma}+\sqrt{k} \mathcal{L}_{k-1,-1}^{\gamma}, & \\
-\sqrt{k} \mathcal{L}_{k-1, m-1}^{\gamma}-\sqrt{k-m} \mathcal{L}_{k, m+1}^{\gamma} & \\
& +\sqrt{k-m+1} \mathcal{L}_{k, m-1}^{\gamma}+\sqrt{k+1} \mathcal{L}_{k+1, m+1}^{\gamma}, & & m<0,
\end{array}\right. \\
& \partial_{x} \mathcal{L}_{k m}^{\gamma}=\frac{\sqrt{\gamma}}{2}\left\{\begin{array}{rlrl}
- & \sqrt{k} \mathcal{L}_{k-1, m+1}^{\gamma}+\sqrt{k+m} \mathcal{L}_{k, m-1}^{\gamma} & \\
& -\sqrt{k+m+1} \mathcal{L}_{k, m+1}^{\gamma}+\sqrt{k+1} \mathcal{L}_{k+1, m-1}^{\gamma}, & & m>0, \\
& \sqrt{k} \mathcal{L}_{k-1,1}^{\gamma}-\sqrt{k+1} \mathcal{L}_{k,-1}^{\gamma} & & \\
& -\sqrt{k+1} \mathcal{L}_{k 1}^{\gamma}-\sqrt{k} \mathcal{L}_{k-1,-1}^{\gamma}, & & m=0, \\
- & \sqrt{k} \mathcal{L}_{k-1, m-1}^{\gamma}+\sqrt{k-m} \mathcal{L}_{k, m+1}^{\gamma} & & \\
& -\sqrt{k-m+1} \mathcal{L}_{k, m-1}^{\gamma}+\sqrt{k+1} \mathcal{L}_{k+1, m+1}^{\gamma}, & & m<0,
\end{array}\right. \\
& \partial_{y} \mathcal{L}_{k m}^{\gamma}=\frac{\mathrm{i} \sqrt{\gamma}}{2}\left\{\begin{array}{rlrl}
\sqrt{k} \mathcal{L}_{k-1, m+1}^{\gamma}+\sqrt{k+m} \mathcal{L}_{k, m-1}^{\gamma} & & \\
& +\sqrt{k+m+1} \mathcal{L}_{k, m+1}^{\gamma}+\sqrt{k+1} \mathcal{L}_{k+1, m-1}^{\gamma}, & & m>0, \\
& \quad+\sqrt{k+1} \mathcal{L}_{k 1}^{\gamma}-\sqrt{k+1} \mathcal{L}_{k,-1}^{\gamma} & & \\
-\sqrt{k} \mathcal{L}_{k-1, m-1}^{\gamma}-\sqrt{k-m} \mathcal{L}_{k, m+1}^{\gamma} & & m=0, \\
& -\sqrt{k-m+1} \mathcal{L}_{k, m-1}^{\gamma}-\sqrt{k+1} \mathcal{L}_{k+1, m+1}^{\gamma}, & & m<0 .
\end{array}\right.
\end{aligned}
$$

Proof We henceforth focus on positive integer numbers $(k, m) \in \mathbb{N}_{\geq 1} \times \mathbb{N}_{\geq 1}$; similar arguments apply to non-positive integers $m \leq 0$ and the special case $k=0$. The amount of technicalities in the proof is significantly reduced by considering $\mathcal{L}_{k m}^{\gamma}: \mathbb{R}^{2} \rightarrow \mathbb{C}:(x, y) \mapsto \mathcal{L}_{k m}^{\gamma}(x, y)$ as a complex function $\mathcal{L}_{k m}^{\gamma}$ : $\mathbb{C} \rightarrow \mathbb{C}: z=x+\mathrm{i} y=r \mathrm{e}^{\mathrm{i} \vartheta} \mapsto \mathcal{L}_{k m}^{\gamma}(z)$; that is, we set $\left(k \in \mathbb{N}, m \in \mathbb{N}_{\geq 1}\right)$

$$
\mathcal{L}_{k m}^{\gamma}(z)=\frac{1}{\sqrt{\pi C_{k}^{m}}} \gamma^{\frac{1}{2}(m+1)} f_{k m}^{\gamma}(z), \quad f_{k m}^{\gamma}(z)=z^{m} \mathrm{e}^{-\frac{1}{2} \gamma|z|^{2}} L_{k}^{m}\left(\gamma|z|^{2}\right)
$$


note that $z^{m}=r^{m} \mathrm{e}^{\mathrm{i} m \vartheta}$.

(i) Applying (8c) and (8e) we obtain $\left((k, m) \in \mathbb{N}_{\geq 1} \times \mathbb{N}_{\geq 1}\right)$

$$
\begin{gathered}
z f_{k m}^{\gamma}=f_{k, m+1}^{\gamma}-f_{k-1, m+1}^{\gamma}, \\
\gamma \bar{z} f_{k m}^{\gamma}=(k+m) f_{k, m-1}^{\gamma}-(k+1) f_{k+1, m-1}^{\gamma} .
\end{gathered}
$$

Employing the relation $x=\frac{1}{2}(z+\bar{z})$ the first statement follows by a brief calculation $\left((k, m) \in \mathbb{N}_{\geq 1} \times \mathbb{N}_{\geq 1}\right)$

$$
\begin{aligned}
x \mathcal{L}_{k m}^{\gamma}= & \frac{1}{2 \sqrt{\pi C_{k}^{m}}} \gamma^{\frac{1}{2}(m+1)}\left(z f_{k m}^{\gamma}+\bar{z} f_{k m}^{\gamma}\right) \\
= & \frac{1}{2 \sqrt{\pi C_{k}^{m}}} \gamma^{\frac{1}{2}(m+1)}\left(f_{k, m+1}^{\gamma}-f_{k-1, m+1}^{\gamma}\right) \\
& \quad+\frac{1}{2 \sqrt{\pi C_{k}^{m}}} \gamma^{\frac{1}{2}(m-1)}\left((k+m) f_{k, m-1}^{\gamma}-(k+1) f_{k+1, m-1}^{\gamma}\right) \\
= & \frac{1}{2 \sqrt{\gamma}}\left(\sqrt{\frac{C_{k}^{m+1}}{C_{k}^{m}}} \mathcal{L}_{k, m+1}^{\gamma}-\sqrt{\frac{C_{k-1}^{m+1}}{C_{k}^{m}}} \mathcal{L}_{k-1, m+1}^{\gamma}\right. \\
& \left.+(k+m) \sqrt{\frac{C_{k}^{m-1}}{C_{k}^{m}}} \mathcal{L}_{k, m-1}^{\gamma}-(k+1) \sqrt{\frac{C_{k+1}^{m-1}}{C_{k}^{m}}} \mathcal{L}_{k+1, m-1}^{\gamma}\right) \\
= & \frac{1}{2 \sqrt{\gamma}}\left(\sqrt{k+m+1} \mathcal{L}_{k, m+1}^{\gamma}-\sqrt{k} \mathcal{L}_{k-1, m+1}^{\gamma}\right. \\
& \left.+\sqrt{k+m} \mathcal{L}_{k, m-1}^{\gamma}-\sqrt{k+1} \mathcal{L}_{k+1, m-1}^{\gamma}\right) ;
\end{aligned}
$$

similar considerations using the identity $y=-\frac{1}{2} \mathrm{i}(z-\bar{z})$ prove the assertion for $y \mathcal{L}_{k m}$.

(ii) In order to deduce the statements for $\partial_{x} \mathcal{L}_{k m}$ and $\partial_{y} \mathcal{L}_{k m}$ we utilize that the differential operators

$$
\partial_{z}=\frac{1}{2}\left(\partial_{x}-\mathrm{i} \partial_{y}\right), \quad \partial_{\bar{z}}=\frac{1}{2}\left(\partial_{x}+\mathrm{i} \partial_{y}\right),
$$

can be applied as if $z$ and $\bar{z}$ were independent variables, see for instance [20, Sect.1.4]. Suitably combining the relations in (8c)-(8f) we thus obtain $\left((k, m) \in \mathbb{N}_{\geq 1} \times \mathbb{N}_{\geq 1}\right)$

$$
\begin{gathered}
\partial_{z} f_{k m}^{\gamma}(z)=\frac{k+1}{2} f_{k+1, m-1}^{\gamma}(z)+\frac{k+m}{2} f_{k, m-1}^{\gamma}(z), \\
\partial_{\bar{z}} f_{k m}^{\gamma}(z)=-\frac{\gamma}{2} f_{k-1, m+1}^{\gamma}(z)-\frac{\gamma}{2} f_{k, m+1}^{\gamma}(z) ;
\end{gathered}
$$

due to $\partial_{x}=\partial_{z}+\partial_{\bar{z}}$ and $\partial_{y}=\mathrm{i}\left(\partial_{z}-\partial_{\bar{z}}\right)$ the assertion follows.

4.2 Functional analytic framework

We recall the basic relations for the complex-valued functions $\mathcal{L}_{k m}^{\gamma}$ introduced in (10); henceforth, we employ the abbreviation $\mathcal{M}=\mathbb{N} \times \mathbb{Z}$. 
Complete orthonormal system. Time-splitting generalized-Laguerre-Fourier pseudo-spectral methods for the full discretization of the two-dimensional Gross-Pitaevskii equation (1)-(2) rely on the fact that the family $\left(\mathcal{L}_{k m}^{\gamma}\right)_{(k, m) \in \mathcal{M}}$ consists of eigenfunctions associated with the densely defined self-adjoint operator $A: D(A) \subset L^{2}\left(\mathbb{R}^{2}\right) \rightarrow L^{2}\left(\mathbb{R}^{2}\right)$ and forms a complete orthonormal system of the Lebesgue space $L^{2}\left(\mathbb{R}^{2}\right)$. In the following, we employ the orthogonality relations

$$
\left(\mathcal{L}_{k m}^{\gamma} \mid \mathcal{L}_{k^{\prime} m^{\prime}}^{\gamma}\right)_{L^{2}}=\delta_{k k^{\prime}} \delta_{m m^{\prime}}, \quad(k, m),\left(k^{\prime}, m^{\prime}\right) \in \mathcal{M},
$$

and the spectral representation for functions $u \in L^{2}\left(\mathbb{R}^{2}\right)$

$$
u=\sum_{(k, m) \in \mathcal{M}} c_{k m}(u) \mathcal{L}_{k m}^{\gamma}, \quad c_{k m}(u)=\left(\mathcal{L}_{k m}^{\gamma} \mid u\right)_{L^{2}},
$$

which by Parseval's identity implies

$$
\|u\|_{L^{2}}^{2}=\sum_{(k, m) \in \mathcal{M}}\left|c_{k m}(u)\right|^{2}, \quad u \in L^{2}\left(\mathbb{R}^{2}\right) .
$$

Moreover, we utilize the eigenvalue relation

$$
\begin{gathered}
A \mathcal{L}_{k m}^{\gamma}=\left(-\frac{1}{2} \Delta+\frac{1}{2} \gamma\left(x^{2}+y^{2}\right)-\Omega L_{z}\right) \mathcal{L}_{k m}^{\gamma}=\lambda_{k m} \mathcal{L}_{k m}^{\gamma}, \\
\lambda_{k m}=\gamma(2 k+|m|+1)-m \Omega, \quad(k, m) \in \mathcal{M} .
\end{gathered}
$$

Fractional power spaces. The basic requirement $|\Omega|<\gamma$ ensures that the linear operator $A: D(A) \subset L^{2}\left(\mathbb{R}^{2}\right) \rightarrow L^{2}\left(\mathbb{R}^{2}\right)$ is positive-definite, see also (2f) and (4). As a consequence, for arbitrary exponents $\alpha \in \mathbb{R}$ the fractional powers $A^{\alpha}: X_{\alpha}=D\left(A^{\alpha}\right) \subset L^{2}\left(\mathbb{R}^{2}\right) \rightarrow L^{2}\left(\mathbb{R}^{2}\right)$, defined by

$$
\begin{gathered}
A^{\alpha} u=\sum_{(k, m) \in \mathcal{M}} c_{k m}(u) \lambda_{k m}^{\alpha} \mathcal{L}_{k m}^{\gamma}, \\
\|u\|_{X_{\alpha}}^{2}=\left\|A^{\alpha} u\right\|_{L^{2}}^{2}=\sum_{(k, m) \in \mathcal{M}}\left|c_{k m}(u)\right|^{2} \lambda_{k m}^{2 \alpha}, \\
X_{\alpha}=\left\{u \in L^{2}\left(\mathbb{R}^{2}\right):\|u\|_{X_{\alpha}}<\infty\right\},
\end{gathered}
$$

are again linear, self-adjoint, and positive-definite operators. The spaces $X_{\alpha}$ are called fractional power spaces associated with the operator $A$; in particular, the relations $X_{0}=L^{2}\left(\mathbb{R}^{2}\right)$ and $X_{1}=D(A)$ hold true. Besides, for exponents $0 \leq \alpha \leq \widetilde{\alpha}$ and $u \in X_{\widetilde{\alpha}}$ the estimate

$$
\|u\|_{X_{\alpha}} \leq \mathcal{C}\|u\|_{X_{\tilde{\alpha}}}
$$

is valid. 
4.3 Estimates in fractional power spaces

In this section, we derive estimates for products of functions in fractional power spaces. A first auxiliary result facilitates to relate estimates with respect to Sobolev-norms to estimates in fractional power spaces.

Lemma 2 For any $\alpha \in \mathbb{N}$ the relation

$$
\|x u\|_{X_{\alpha}}+\|y u\|_{X_{\alpha}}+\left\|\partial_{x} u\right\|_{X_{\alpha}}+\left\|\partial_{y} u\right\|_{X_{\alpha}} \leq \mathcal{C}\|u\|_{X_{\alpha+\frac{1}{2}}}, \quad u \in X_{\alpha+\frac{1}{2}},
$$

holds with constant $\mathcal{C}>0$ depending in particular on $\gamma$ and $\Omega$.

Proof In order to treat all cases simultaneously, we denote by $F \in\left\{x, y, \partial_{x}, \partial_{y}\right\}$ a multiplication or differentiation operator, respectively. Lemma 1 implies that $F \mathcal{L}_{k m}^{\gamma}$ can be represented in the form

$$
F \mathcal{L}_{k m}^{\gamma}=\sum_{\substack{k^{\prime}=k-1, k, k+1 \\ m^{\prime}=m-1, m+1}} a_{k^{\prime} m^{\prime}}^{k m} \mathcal{L}_{k^{\prime} m^{\prime}}^{\gamma}, \quad(k, m) \in \mathcal{M},
$$

where only certain coefficients with neighboring indices are nonzero. Conversely, for $\left(k^{\prime}, m^{\prime}\right) \in \mathcal{M}$ given $a_{k^{\prime} m^{\prime}}^{k m} \neq 0$ holds for $k \in\left\{k^{\prime}-1, k^{\prime}, k^{\prime}+1\right\}$ and $m \in\left\{m^{\prime}-1, m^{\prime}+1\right\}$. Consequently, we obtain

$$
\begin{aligned}
A^{\alpha} F u & =\sum_{(k, m) \in \mathcal{M}} c_{k m}(u) A^{\alpha} F \mathcal{L}_{k m}^{\gamma} \\
& =\sum_{\left(k^{\prime}, m^{\prime}\right) \in \mathcal{M}} \sum_{\substack{k=k^{\prime}-1, k^{\prime}, k^{\prime}+1 \\
m=m^{\prime}-1, m^{\prime}+1}} c_{k m}(u) a_{k^{\prime} m^{\prime}}^{k m} \lambda_{k^{\prime} m^{\prime}}^{\alpha} \mathcal{L}_{k^{\prime} m^{\prime}}^{\gamma} .
\end{aligned}
$$

In order to relate the coefficients $a_{k^{\prime} m^{\prime}}^{k m}$ with explicit values specified in Lemma 1 to the eigenvalue $\lambda_{k m}$, we employ the estimate

$$
\begin{aligned}
\lambda_{k m} & =\gamma(2 k+|m|+1)-\Omega m \geq \gamma(2 k+1)+(\gamma-|\Omega|)|m| \\
& \geq(\gamma-|\Omega|)(k+|m|+1),
\end{aligned}
$$

recalling that $|\Omega|<\gamma$ by assumption, such that

$$
\left|a_{k^{\prime} m^{\prime}}^{k m}\right|^{2} \leq \max \left\{\gamma, \gamma^{-1}\right\}(k+|m|+1) \leq \frac{1}{\gamma-|\Omega|} \max \left\{\gamma, \gamma^{-1}\right\} \lambda_{k m} \leq \mathcal{C} \lambda_{k m} .
$$

By means of the arithmetic-geometric mean inequality the bound

$$
\begin{aligned}
\Re\left(z_{j} \bar{z}_{k}\right) & =\Re\left(\left(x_{j}+\mathrm{i} y_{j}\right)\left(x_{k}-\mathrm{i} y_{k}\right)\right)=x_{j} x_{k}+y_{j} y_{k} \leq \sqrt{x_{j}^{2} x_{k}^{2}}+\sqrt{y_{j}^{2} y_{k}^{2}} \\
& \leq \frac{1}{2}\left(x_{j}^{2}+x_{k}^{2}+y_{j}^{2}+y_{k}^{2}\right)=\frac{1}{2}\left(\left|z_{j}\right|^{2}+\left|z_{k}\right|^{2}\right)
\end{aligned}
$$

follows, which further implies

$$
\left|\sum_{j=1}^{J} z_{j}\right|^{2}=\sum_{j=1}^{J}\left|z_{j}\right|^{2}+2 \sum_{\substack{j, k=1 \\ k>j}}^{J} \Re\left(z_{j} \bar{z}_{k}\right) \leq J \sum_{j=1}^{J}\left|z_{j}\right|^{2}, \quad\left(z_{1}, \ldots, z_{J}\right) \in \mathbb{C}^{J} .
$$


Making use of the fact that $\lambda_{k^{\prime} m^{\prime}} \leq \mathcal{C} \lambda_{k m}$ holds for all $k^{\prime} \in\{k-1, k, k+1\}$ and $m^{\prime} \in\{m-1, m+1\}$ we thus obtain

$$
\begin{aligned}
\left\|A^{\alpha} F u\right\|_{X_{0}}^{2} & =\sum_{\left(k^{\prime}, m^{\prime}\right) \in \mathcal{M}} \lambda_{k^{\prime} m^{\prime}}^{2 \alpha}\left|\sum_{\substack{k=k^{\prime}-1, k^{\prime}, k^{\prime}+1 \\
m=m^{\prime}-1, m^{\prime}+1}} c_{k m}(u) a_{k^{\prime} m^{\prime}}^{k m}\right|^{2} \\
& \leq \mathcal{C} \sum_{\substack{\left(k^{\prime}, m^{\prime}\right) \in \mathcal{M} \\
\lambda_{k^{\prime} m^{\prime}}^{2 \alpha}}} \sum_{\substack{k=k^{\prime}-1, k^{\prime}, k^{\prime}+1 \\
m=m^{\prime}-1, m^{\prime}+1}}\left|c_{k m}(u)\right|^{2} \lambda_{k m} \\
& =\mathcal{C} \sum_{(k, m) \in \mathcal{M}}\left|c_{k m}(u)\right|^{2} \lambda_{k m} \sum_{\substack{k^{\prime}=k-1, k, k+1 \\
m^{\prime}=m-1, m+1}} \lambda_{k^{\prime} m^{\prime}}^{2 \alpha} \\
& =\mathcal{C} \sum_{(k, m) \in \mathcal{M}}\left|c_{k m}(u)\right|^{2} \lambda_{k m}^{2 \alpha+1} .
\end{aligned}
$$

This further yields the estimate

$$
\begin{aligned}
\|F u\|_{X_{\alpha}}^{2} & =\left\|A^{\alpha} F u\right\|_{X_{0}}^{2} \leq \mathcal{C} \sum_{(k, m) \in \mathcal{M}}\left|c_{k m}(u)\right|^{2} \lambda_{k m}^{2 \alpha+1}=\mathcal{C}\left\|A^{\alpha+\frac{1}{2}} u\right\|_{X_{0}}^{2} \\
& =\mathcal{C}\|u\|_{X_{\alpha+\frac{1}{2}}}^{2}
\end{aligned}
$$

and proves the stated result.

The following auxiliary result implies that fractional power spaces form a normed algebra for positive integer exponents; its proof relies on Lemma 2 and the well-known Sobolev imbedding theorem

$$
\|u\|_{L^{\infty}} \leq \mathcal{C}\|u\|_{H^{2}}, \quad u \in H^{2}\left(\mathbb{R}^{2}\right) .
$$

Lemma 3 For any $\alpha \in \mathbb{N}_{\geq 1}$ the following estimates hold

$$
\begin{gathered}
\|u\|_{L^{\infty}} \leq \mathcal{C}\|u\|_{H^{2}} \leq \mathcal{C}\|u\|_{X_{\alpha}}, \quad u \in X_{\alpha} \\
\|u v\|_{X_{0}} \leq \mathcal{C}\|u\|_{X_{0}}\|v\|_{X_{\alpha}}, \quad u \in X_{0}, \quad v \in X_{\alpha} \\
\|u v\|_{X_{\alpha}} \leq \mathcal{C}\|u\|_{X_{\alpha}}\|v\|_{X_{\alpha}}, \quad u, v \in X_{\alpha}
\end{gathered}
$$

Proof The first estimate follows by means of the Sobolev imbedding theorem and Lemma 2 , since $\left(u \in X_{\alpha}\right)$

$$
\|u\|_{L^{\infty}}^{2} \leq \mathcal{C}\|u\|_{H^{2}}^{2}=\mathcal{C}\left(\|u\|_{L^{2}}^{2}+\left\|\partial_{x}^{2} u\right\|_{L^{2}}^{2}+\left\|\partial_{y}^{2} u\right\|_{L^{2}}^{2}\right) \leq \mathcal{C}\|u\|_{X_{\alpha}}^{2} ;
$$

this immediately implies the second bound $\left(u \in X_{0}, v \in X_{\alpha}\right)$

$$
\|u v\|_{X_{0}}=\|u v\|_{L^{2}} \leq\|u\|_{L^{2}}\|v\|_{L^{\infty}} \leq \mathcal{C}\|u\|_{X_{0}}\|v\|_{X_{\alpha}} .
$$

In order to deduce the third estimate, we employ the special structure of the linear operator

$$
A=-\frac{1}{2}\left(\partial_{x}^{2}+\partial_{y}^{2}\right)+\frac{1}{2} \gamma\left(x^{2}+y^{2}\right)+\mathrm{i} \Omega\left(x \partial_{y}-y \partial_{x}\right),
$$


see also (2f). For exponents $\alpha \in \mathbb{N}_{\geq 1}$ the expression $A^{\alpha}(u v)$ is determined by means of the differentiation rule of Leibnitz. The arising terms are then estimated by suitably balancing the terms involved. For instance, we employ the following relation $\left(u, v \in X_{\alpha}\right)$

$$
\begin{aligned}
\left\|\partial_{x}^{2 \alpha-j} u \partial_{x}^{j} v\right\|_{L^{2}} & \leq\left\|\partial_{x}^{2 \alpha-j} u\right\|_{L^{2}}\left\|\partial_{x}^{j} v\right\|_{L^{\infty}} \leq \mathcal{C}\|u\|_{X_{\alpha-\frac{j}{2}}}\|v\|_{X_{\frac{j}{2}+1}} \\
& \leq \mathcal{C}\|u\|_{X_{\alpha}}\|v\|_{X_{\alpha}},
\end{aligned}
$$

which remains valid for $0 \leq j \leq \alpha$ if $\alpha \in \mathbb{N}_{\geq 2}$; for the special case $\alpha=1$ we instead apply the Cauchy-Schwarz inequality and the continuous imbedding $H^{1}\left(\mathbb{R}^{2}\right) \subset L^{4}\left(\mathbb{R}^{2}\right)$, see [2], to obtain

$$
\begin{aligned}
\left\|\partial_{x} u \partial_{x} v\right\|_{L^{2}} & \leq\left\|\partial_{x} u\right\|_{L^{4}}\left\|\partial_{x} v\right\|_{L^{4}} \leq \mathcal{C}\left\|\partial_{x} u\right\|_{H^{1}}\left\|\partial_{x} v\right\|_{H^{1}} \leq \mathcal{C}\|u\|_{H^{2}}\|v\|_{H^{2}} \\
& \leq \mathcal{C}\|u\|_{X_{1}}\|v\|_{X_{1}} .
\end{aligned}
$$

In perspective of the extension of the auxiliary results to three space dimensions indicated in Section 5.3 we note that the continuous imbeddings $H^{2}\left(\mathbb{R}^{3}\right) \subset L^{\infty}\left(\mathbb{R}^{3}\right)$ and $H^{1}\left(\mathbb{R}^{3}\right) \subset L^{4}\left(\mathbb{R}^{3}\right)$ are valid.

\subsection{Sobolev-type inequalities}

In this section, we derive a Sobolev-type inequality that is needed in the proof of Lemma 6 to estimate the maximum value of a function defined on a curved rectangular domain; prerequisites for its proof are provided by the following result.

Lemma 4 (i) (See also [21]). For any $u \in H^{1}(a, b)$ with $a<b$ the relation

$$
\max _{x \in[a, b]}|u(x)| \leq \frac{1}{b-a}\|u\|_{L^{1}}+\left\|\frac{\mathrm{d}}{\mathrm{d} x} u\right\|_{L^{1}} \leq \frac{1}{\sqrt{b-a}}\|u\|_{L^{2}}+\sqrt{b-a}\left\|\frac{\mathrm{d}}{\mathrm{d} x} u\right\|_{L^{2}}
$$

is valid.

(ii) Let $\Omega=(a, b) \times(c, d)$ with $a<b$ and $c<d$. For any $u \in H^{2}\left(\Omega_{1}\right)$ such that $\Omega_{1} \supset \bar{\Omega}$ is a bounded Lipschitz domain the estimate

$$
\begin{aligned}
\max _{(x, y) \in \bar{\Omega}}|u(x, y)| \leq & \frac{1}{(b-a)(d-c)}\|u\|_{L^{1}}+\frac{1}{d-c}\left\|\partial_{x} u\right\|_{L^{1}}+\frac{1}{b-a}\left\|\partial_{y} u\right\|_{L^{1}} \\
& \quad+2\left\|\partial_{x y} u\right\|_{L^{1}} \\
\leq & \frac{1}{\sqrt{(b-a)(d-c)}}\|u\|_{L^{2}}+\sqrt{\frac{b-a}{d-c}}\left\|\partial_{x} u\right\|_{L^{2}} \\
& \quad+\sqrt{\frac{d-c}{b-a}}\left\|\partial_{y} u\right\|_{L^{2}}+2 \sqrt{(b-a)(d-c)}\left\|\partial_{x y} u\right\|_{L^{2}}
\end{aligned}
$$

with respect to the norms in $L^{1}(\Omega)$ and $L^{2}(\Omega)$ holds. 
Proof For completeness and as the derivation of statement (ii) relies on arguments used in the derivation of statement (i), we include the proof of the first Sobolev inequality.

(i) For any function $u \in H^{1}(a, b)$ the Sobolev imbedding theorem implies $u \in C[a, b]$; indeed, for all $x_{1}, x_{2} \in[a, b]$ with $x_{1}<x_{2}$ the estimate

$$
\left|u\left(x_{1}\right)-u\left(x_{2}\right)\right| \leq \int_{x_{1}}^{x_{2}}\left|\frac{\mathrm{d}}{\mathrm{d} x} u(x)\right| \mathrm{d} x \leq \sqrt{x_{2}-x_{1}}\left\|\frac{\mathrm{d}}{\mathrm{d} x} u\right\|_{L^{2}} \leq \sqrt{x_{2}-x_{1}}\|u\|_{H^{1}}
$$

holds. Consequently, there exists $x_{*} \in[a, b]$ such that

$$
\left|u\left(x_{*}\right)\right|=\min _{x \in[a, b]}|u(x)| \leq \frac{1}{b-a} \int_{a}^{b}|u(x)| \mathrm{d} x=\frac{1}{b-a}\|u\|_{L^{1}},
$$

and by the triangular inequality the estimate $(x \in[a, b])$

$$
|u(x)|-\left|u\left(x_{*}\right)\right| \leq\left|u(x)-u\left(x_{*}\right)\right| \leq \int_{a}^{b}\left|\frac{\mathrm{d}}{\mathrm{d} x} u(x)\right| \mathrm{d} x=\left\|\frac{\mathrm{d}}{\mathrm{d} x} u\right\|_{L^{1}}
$$

follows. Together with the Cauchy-Schwarz inequality this proves the first statement.

(ii) Let $\left(x_{1}, y_{1}\right),\left(x_{2}, y_{2}\right) \in \bar{\Omega}=[a, b] \times[c, d]$. For any function $u \in H^{2}\left(\Omega_{1}\right)$ the Sobolev imbedding theorem ensures $u \in C\left(\Omega_{1}\right)$; setting

$$
v(\cdot)=\int_{y_{1}}^{y_{2}} \partial_{y} u(\cdot, y) \mathrm{d} y \in H^{1}(a, b),
$$

statement (i) thus implies

$$
\begin{aligned}
& \left|u\left(x_{1}, y_{1}\right)-u\left(x_{1}, y_{2}\right)\right|=\left|v\left(x_{1}\right)\right| \leq \max _{x \in[a, b]}|v(x)| \\
& \quad \leq \frac{1}{b-a} \int_{a}^{b}|v(x)| \mathrm{d} x+\int_{a}^{b}\left|\frac{\mathrm{d}}{\mathrm{d} x} v(x)\right| \mathrm{d} x \\
& \quad \leq \frac{1}{b-a} \int_{a}^{b} \int_{c}^{d}\left|\partial_{y} u(x, y)\right| \mathrm{d} y \mathrm{~d} x+\int_{a}^{b} \int_{c}^{d}\left|\partial_{x y} u(x, y)\right| \mathrm{d} y \mathrm{~d} x \\
& \quad=\frac{1}{b-a}\left\|\partial_{y} u\right\|_{L^{1}}+\left\|\partial_{x y} u\right\|_{L^{1}},
\end{aligned}
$$

where the upper bound is independent of $y_{1}, y_{2} \in[c, d]$; analogously, we obtain

$$
\left|u\left(x_{1}, y_{2}\right)-u\left(x_{2}, y_{2}\right)\right| \leq \frac{1}{d-c}\left\|\partial_{x} u\right\|_{L^{1}}+\left\|\partial_{x y} u\right\|_{L^{1}} \text {. }
$$

As a consequence, the estimate

$$
\begin{aligned}
\left|u\left(x_{1}, y_{1}\right)-u\left(x_{2}, y_{2}\right)\right| & \leq\left|u\left(x_{1}, y_{1}\right)-u\left(x_{1}, y_{2}\right)\right|+\left|u\left(x_{1}, y_{2}\right)-u\left(x_{2}, y_{2}\right)\right| \\
& \leq \frac{1}{d-c}\left\|\partial_{x} u\right\|_{L^{1}}+\frac{1}{b-a}\left\|\partial_{y} u\right\|_{L^{1}}+2\left\|\partial_{x y} u\right\|_{L^{1}}
\end{aligned}
$$

follows. Similarly to before, we use that the minimum value satisfies

$$
\left|u\left(x_{*}, y_{*}\right)\right|=\min _{(x, y) \in \bar{\Omega}}|u(x, y)| \leq \frac{1}{(b-a)(d-c)}\|u\|_{L^{1}} .
$$

Setting $\left(x_{2}, y_{2}\right)=\left(x_{*}, y_{*}\right)$ and applying the Cauchy-Schwarz inequality proves the assertion. 
The following result provides a Sobolev-type inequality on a curved rectangle; such a domain naturally arises in connection with polar coordinates.

Lemma 5 For $0<r_{a}<r_{b}, \vartheta_{a}<\vartheta_{b}$, and $\vartheta_{b}-\vartheta_{a} \leq 2 \pi$ set

$$
R=\left\{(x, y)=(r \cos \vartheta, r \sin \vartheta): r \in\left(r_{a}, r_{b}\right), \vartheta \in\left(\vartheta_{a}, \vartheta_{b}\right)\right\} .
$$

Then for any $u \in H^{2}\left(\mathbb{R}^{2}\right)$ the estimate

$$
\begin{aligned}
& \max _{(x, y) \in \bar{R}}|u(x, y)| \leq c_{0}\|u\|_{L^{2}}+\left(c_{11}+c_{12}+c_{13}\right)\left(\left\|\partial_{x} u\right\|_{L^{2}}+\left\|\partial_{y} u\right\|_{L^{2}}\right) \\
& +c_{2}\left(\left\|\partial_{x}^{2} u\right\|_{L^{2}}+\left\|\partial_{y}^{2} u\right\|_{L^{2}}+\left\|\partial_{x y} u\right\|_{L^{2}}\right), \\
& c_{0}=\frac{1}{\sqrt{\operatorname{Vol} R}}=\frac{1}{\sqrt{\frac{1}{2}\left(r_{b}^{2}-r_{a}^{2}\right)\left(\vartheta_{b}-\vartheta_{a}\right)}}, \\
& c_{11}=\frac{1}{\sqrt{\vartheta_{b}-\vartheta_{a}}} \sqrt{\ln \frac{r_{b}}{r_{a}}} \leq \frac{1}{\sqrt{\vartheta_{b}-\vartheta_{a}}} \sqrt{\frac{r_{b}^{2}-r_{a}^{2}}{2 r_{a}^{2}}}, \quad c_{12}=\sqrt{\frac{\left(\vartheta_{b}-\vartheta_{a}\right)\left(r_{a}+r_{b}\right)}{2\left(r_{b}-r_{a}\right)}}, \\
& c_{13}=2 \sqrt{\left(\vartheta_{b}-\vartheta_{a}\right) \ln \frac{r_{b}}{r_{a}}} \leq 2 \sqrt{\vartheta_{b}-\vartheta_{a}} \sqrt{\frac{r_{b}^{2}-r_{a}^{2}}{2 r_{a}^{2}}} \\
& c_{2}=2 \sqrt{\mathrm{Vol} R}=\sqrt{2\left(r_{b}^{2}-r_{a}^{2}\right)\left(\vartheta_{b}-\vartheta_{a}\right)},
\end{aligned}
$$

is valid with respect to the norm in $L^{2}(R)$.

Proof The derivation of the statement relies on a transformation from Cartesian coordinates $(x, y) \in \mathbb{R}^{2}$ to polar coordinates $(r, \vartheta) \in[0, \infty) \times[0,2 \pi)$ and an application of the Sobolev inequality provided by Lemma 4. For this purpose, it is useful to introduce the associated rectangle

$$
\widetilde{R}=\left\{(r, \vartheta): r \in\left(r_{a}, r_{b}\right), \vartheta \in\left(\vartheta_{a}, \vartheta_{b}\right)\right\}
$$

besides, for some bounded Lipschitz domain $\widetilde{R}_{1} \supseteq \widetilde{R}$ we define $\widetilde{u} \in H^{2}\left(\widetilde{R}_{1}\right)$ by

$$
\widetilde{u}(r, \vartheta)=u(r \cos \vartheta, r \sin \vartheta)=u(x, y), \quad(r, \vartheta) \in \widetilde{R}_{1} .
$$

For all $\left(x_{1}, y_{1}\right),\left(x_{2}, y_{2}\right) \in \bar{R}$ relation (13) yields the bound

$$
\begin{aligned}
& \left|u\left(x_{1}, y_{1}\right)-u\left(x_{2}, y_{2}\right)\right|=\left|\widetilde{u}\left(r_{1}, \vartheta_{1}\right)-\widetilde{u}\left(r_{2}, \vartheta_{2}\right)\right| \\
& \quad \leq \frac{1}{\vartheta_{b}-\vartheta_{a}}\left\|\partial_{r} \widetilde{u}\right\|_{L^{1}(\widetilde{R})}+\frac{1}{r_{b}-r_{a}}\left\|\partial_{\vartheta} \widetilde{u}\right\|_{L^{1}(\widetilde{R})}+2\left\|\partial_{r \vartheta} \widetilde{u}\right\|_{L^{1}(\widetilde{R})} .
\end{aligned}
$$

We next deduce estimates for the quantities arising on the right-hand side; the relations

$$
\begin{aligned}
\left|\partial_{r} \widetilde{u}(r, \vartheta)\right| & =\left|\cos \vartheta \partial_{x} u(x, y)+\sin \vartheta \partial_{y} u(x, y)\right| \leq\left|\partial_{x} u(x, y)\right|+\left|\partial_{y} u(x, y)\right| \\
\left|\partial_{\vartheta} \widetilde{u}(r, \vartheta)\right|= & r\left|-\sin \vartheta \partial_{x} u(x, y)+\cos \vartheta \partial_{y} u(x, y)\right| \\
\leq & r\left(\left|\partial_{x} u(x, y)\right|+\left|\partial_{y} u(x, y)\right|\right), \\
\left|\partial_{r \vartheta} \widetilde{u}(r, \vartheta)\right|=\mid & -\sin \vartheta \partial_{x} u(x, y)+\cos \vartheta \partial_{y} u(x, y)-r \sin \vartheta \cos \vartheta \partial_{x}^{2} u(x, y) \\
& \quad+r \sin \vartheta \cos \vartheta \partial_{y}^{2} u(x, y)+r\left(\cos ^{2} \vartheta-\sin ^{2} \vartheta\right) \partial_{x y} u(x, y) \mid \\
\leq & \left|\partial_{x} u(x, y)\right|+\left|\partial_{y} u(x, y)\right| \\
& \quad+r\left(\left|\partial_{x}^{2} u(x, y)\right|+\left|\partial_{y}^{2} u(x, y)\right|+\left|\partial_{x y} u(x, y)\right|\right),
\end{aligned}
$$


together with suitable integral transformations and the Cauchy-Schwarz inequality imply

$$
\begin{aligned}
\left\|\partial_{r} \widetilde{u}\right\|_{L^{1}(\widetilde{R})} & =\int_{r_{a}}^{r_{b}} \int_{\vartheta_{a}}^{\vartheta_{b}}\left|\partial_{r} \widetilde{u}(r, \vartheta)\right| \mathrm{d} \vartheta \mathrm{d} r \\
& \leq \int_{R} \frac{1}{\sqrt{x^{2}+y^{2}}}\left|\partial_{x} u(x, y)\right| \mathrm{d}(x, y)+\int_{R} \frac{1}{\sqrt{x^{2}+y^{2}}}\left|\partial_{y} u(x, y)\right| \mathrm{d}(x, y) \\
& \leq\left(\int_{R} \frac{1}{x^{2}+y^{2}} \mathrm{~d} x \mathrm{~d} y\right)^{\frac{1}{2}}\left(\left\|\partial_{x} u\right\|_{L^{2}(R)}+\left\|\partial_{y} u\right\|_{L^{2}(R)}\right) \\
& =\left(\int_{r_{a}}^{r_{b}} \int_{\vartheta_{a}}^{\vartheta_{b}} \frac{1}{r} \mathrm{~d} \vartheta \mathrm{d} r\right)^{\frac{1}{2}}\left(\left\|\partial_{x} u\right\|_{L^{2}(R)}+\left\|\partial_{y} u\right\|_{L^{2}(R)}\right) \\
& =\sqrt{\left(\vartheta_{b}-\vartheta_{a}\right) \ln \frac{r_{b}}{r_{a}}}\left(\left\|\partial_{x} u\right\|_{L^{2}(R)}+\left\|\partial_{y} u\right\|_{L^{2}(R)}\right)
\end{aligned}
$$

and, in a similar manner, we obtain

$$
\begin{aligned}
\left\|\partial_{\vartheta} \widetilde{u}\right\|_{L^{1}(\widetilde{R})} \leq & \sqrt{\frac{1}{2}\left(\vartheta_{b}-\vartheta_{a}\right)\left(r_{b}^{2}-r_{a}^{2}\right)}\left(\left\|\partial_{x} u\right\|_{L^{2}(R)}+\left\|\partial_{y} u\right\|_{L^{2}(R)}\right) \\
\left\|\partial_{r \vartheta} \widetilde{u}\right\|_{L^{1}(\widetilde{R})} \leq & \sqrt{\left(\vartheta_{b}-\vartheta_{a}\right) \ln \frac{r_{b}}{r_{a}}}\left(\left\|\partial_{x} u\right\|_{L^{2}(R)}+\left\|\partial_{y} u\right\|_{L^{2}(R)}\right) \\
& +\sqrt{\frac{1}{2}\left(\vartheta_{b}-\vartheta_{a}\right)\left(r_{b}^{2}-r_{a}^{2}\right)}\left(\left\|\partial_{x}^{2} u\right\|_{L^{2}(R)}+\left\|\partial_{y}^{2} u\right\|_{L^{2}(R)}\right. \\
& \left.+\left\|\partial_{x y} u\right\|_{L^{2}(R)}\right)
\end{aligned}
$$

we further note that the estimate

$$
\ln \frac{r_{b}}{r_{a}}=\frac{1}{2}\left(\ln r_{b}^{2}-\ln r_{a}^{2}\right)=\frac{1}{2} \int_{r_{a}^{2}}^{r_{b}^{2}} \frac{1}{r} \mathrm{~d} r \leq \frac{1}{2} \frac{r_{b}^{2}-r_{a}^{2}}{r_{a}^{2}}
$$

holds. Inserting the above bounds thus yields

$$
\begin{aligned}
\left|u\left(x_{1}, y_{1}\right)-u\left(x_{2}, y_{2}\right)\right| \leq( & \left.c_{11}+c_{12}+c_{13}\right)\left(\left\|\partial_{x} u\right\|_{L^{2}(R)}+\left\|\partial_{y} u\right\|_{L^{2}(R)}\right) \\
& +c_{2}\left(\left\|\partial_{x}^{2} u\right\|_{L^{2}(R)}+\left\|\partial_{y}^{2} u\right\|_{L^{2}(R)}+\left\|\partial_{x y} u\right\|_{L^{2}(R)}\right) .
\end{aligned}
$$

As in the proof of Lemma 4 we use that the minimum value satisfies

$$
\left|u\left(x_{*}, y_{*}\right)\right|=\min _{(x, y) \in \bar{R}}|u(x, y)| \leq \frac{1}{\operatorname{Vol} R}\|u\|_{L^{1}(R)} \leq \frac{1}{\sqrt{\operatorname{Vol} R}}\|u\|_{L^{2}(R)} ;
$$

setting $\left(x_{2}, y_{2}\right)=\left(x_{*}, y_{*}\right)$ proves the assertion.

4.5 Estimate in a discrete $L^{2}$-norm

In the following, we deduce a bound with respect to a discrete $L^{2}$-norm related to Gauß-Laguerre quadrature and weights, needed for the estimation of the generalized-Laguerre-Fourier spectral interpolant. For this purpose, we first study the asymptotical distribution of the Gauß-Laguerre quadrature nodes and weights. 
Gauß-Laguerre quadrature nodes and weights. For the (standard) Laguerre polynomial of degree $\kappa \in \mathbb{N}_{\geq 1}$

$$
L_{\kappa}(r)=L_{\kappa}^{0}(r)=\frac{1}{\kappa !} \mathrm{e}^{r} \frac{\mathrm{d}^{\kappa}}{\mathrm{d} r^{\kappa}}\left(\mathrm{e}^{-r} r^{\kappa}\right), \quad r \in \mathbb{R},
$$

the zeros and corresponding weights associated with the Gauß-Laguerre quadrature formula of order $2 \kappa$ are henceforth denoted by

$$
\left(\varrho_{j \kappa}, \omega_{j \kappa}\right)_{j=0}^{\kappa-1},
$$

see also (8) for the definition of the generalized-Laguerre polynomials and [11] for the numerical computation of the quadrature nodes and weights. That is, for a (regular) function $f: \mathbb{R}_{\geq 0} \rightarrow \mathbb{R}$ an approximation to the integral over the positive real line is obtained by

$$
\sum_{j=0}^{\kappa-1} \omega_{j \kappa} f\left(\varrho_{j \kappa}\right) \approx \int_{0}^{\infty} \mathrm{e}^{-\varrho} f(\varrho) \mathrm{d} \varrho,
$$

with exact quadrature for polynomials of degree at most $2 \kappa-1$.

Asymptotical distribution. The smallest zero and the largest zero of the $\kappa$-th Laguerre polynomial satisfy the relations

$$
\mathcal{C}_{1} \kappa^{-1} \leq \varrho_{0 \kappa} \leq \mathcal{C}_{2} \kappa^{-1}, \quad \varrho_{\kappa-1, \kappa} \leq 4 \kappa,
$$

with constants $\mathcal{C}_{1}, \mathcal{C}_{2}>0$ independent of $\kappa$, see [15, Thm $\left.1.4(1.22)\right]$ and [19, $\S 18.16 .13]$. Following [15, Eq. (1.18)] we introduce the function

$$
\varphi_{\kappa}:[0,4 \kappa] \longrightarrow \mathbb{R}: r \longmapsto \varphi_{\kappa}(r)=\frac{\sqrt{r+4 \kappa^{-1}}(8 \kappa-r)}{\kappa \sqrt{4 \kappa+4 \kappa^{\frac{1}{3}}-r}} .
$$

For $\kappa \geq \kappa_{0}$ the quadrature weights satisfy the relation $(j=0,1, \ldots, \kappa-1)$

$$
\mathcal{C}_{1} \varphi_{\kappa}\left(\varrho_{j \kappa}\right) \leq \omega_{j \kappa} \mathrm{e}^{\varrho_{j \kappa}} \leq \mathcal{C}_{2} \varphi_{\kappa}\left(\varrho_{j \kappa}\right)
$$

with constants $\mathcal{C}_{1}, \mathcal{C}_{2}$ independent of $\kappa$ and $j$, see [15, Thm 1.3 (1.19)]; furthermore, for $\kappa \geq 1$ the relation $(j=1,2, \ldots, \kappa-1)$

$$
\mathcal{C}_{1} \varphi_{\kappa}\left(\varrho_{j \kappa}\right) \leq \varrho_{j \kappa}-\varrho_{j-1, \kappa} \leq \mathcal{C}_{2} \varphi_{\kappa}\left(\varrho_{j \kappa}\right)
$$

holds with constants $\mathcal{C}_{1}, \mathcal{C}_{2}$ independent of $\kappa$ and $j$, see [16, Theorem 1.4]. By elementary calculus it follows that $\varphi_{\kappa}$ has no local extremum in $(0,4 \kappa)$ for all $\kappa \geq 2$; hence the minimum and maximum values are attained at the boundary points $r=0$ and $r=4 \kappa$, respectively, such that

$$
\begin{array}{cl}
\varphi_{\kappa}(r) \geq \varphi_{\kappa}(0)=\frac{8}{\sqrt{\kappa^{2}+\kappa^{\frac{4}{3}}}} \geq 4 \sqrt{2} \kappa^{-1}, & r \in[0,4 \kappa], \\
\varphi_{\kappa}(r) \leq \varphi_{\kappa}(4 \kappa)=4 \frac{\sqrt{\kappa+\kappa^{-1}}}{\kappa^{1 / 6}} \leq 4 \sqrt{2} \kappa^{\frac{1}{3}}, \quad r \in[0,4 \kappa] .
\end{array}
$$


Moreover, due to the fact that the function $r \mapsto \frac{(8 \kappa-r)^{2}}{4 \kappa+4 \kappa^{\frac{1}{3}}-r}$ has no local maximum in $(0,4 \kappa)$ and thus attains its maximum at $r=0$ or $r=4 \kappa$, the estimate

$$
\begin{aligned}
\frac{1}{r}\left(\varphi_{\kappa}(r)\right)^{2} & =\frac{\left(1+\frac{4}{\kappa r}\right)(8 \kappa-r)^{2}}{\kappa^{2}\left(4 \kappa+4 \kappa^{\frac{1}{3}}-r\right)} \leq \mathcal{C} \frac{(8 \kappa-r)^{2}}{\kappa^{2}\left(4 \kappa+4 \kappa^{\frac{1}{3}}-r\right)} \\
& \leq \mathcal{C} \kappa^{-\frac{1}{3}}, \quad \mathcal{C} \kappa^{-1} \leq r \leq 4 \kappa
\end{aligned}
$$

is valid, and by means of relation (16a) the bound

$$
\varphi_{\kappa}\left(\varrho_{0 \kappa}\right) \leq \frac{8 \kappa \sqrt{\left(\mathcal{C}_{2}+4\right) \kappa^{-1}}}{\kappa \sqrt{4 \kappa+4 \kappa^{\frac{1}{3}}-\mathcal{C}_{2} \kappa^{-1}}} \leq \mathcal{C} \frac{\kappa^{-\frac{1}{2}}}{\kappa^{\frac{1}{2}}}=\mathcal{C} \kappa^{-1}
$$

follows.

Discrete $L^{2}$-norm. In regard to $(9)$ and (15) we introduce the scaled GaußLaguerre nodes and weights $(j=0,1, \ldots, \kappa-1)$

$$
r_{j \kappa}=\sqrt{\frac{\varrho_{j \kappa}}{\gamma}}, \quad w_{j \kappa}=\frac{\pi}{\gamma} \omega_{j \kappa} \mathrm{e}^{\varrho_{j \kappa}}
$$

which yields the quadrature approximation

$$
\begin{aligned}
& \sum_{j=0}^{\kappa-1} w_{j \kappa} g\left(r_{j \kappa}\right)=\frac{\pi}{\gamma} \sum_{j=0}^{\kappa-1} \omega_{j \kappa} \mathrm{e}^{\varrho_{j \kappa}} g\left(\sqrt{\frac{\varrho_{j \kappa}}{\gamma}}\right) \\
& \approx \frac{\pi}{\gamma} \int_{0}^{\infty} g\left(\sqrt{\frac{\varrho}{\gamma}}\right) \mathrm{d} \varrho=2 \pi \int_{0}^{\infty} r g(r) \mathrm{d} r,
\end{aligned}
$$

see also [4, Eq. (2.29)]; furthermore, we consider the equidistant nodes associated with the trapezoidal rule $\left(\mu \in \mathbb{N}_{\geq 1}, s=0,1, \ldots, \mu-1\right)$

$$
\vartheta_{s \mu}=\frac{2 \pi s}{\mu} \text {. }
$$

The iterated quadrature formula then leads to the approximation

$$
\frac{1}{\mu} \sum_{j=0}^{\kappa-1} \sum_{\mu=0}^{\mu-1} w_{j \kappa} g\left(r_{j \kappa}, \vartheta_{s \mu}\right) \approx \int_{0}^{\infty} \int_{0}^{2 \pi} r g(r, \vartheta) \mathrm{d} \vartheta \mathrm{d} r
$$

we note that the weight associated with the trapezoidal rule is reflected in the prefactor $\frac{1}{\mu}$ and the scaling of $w_{j \kappa}$. Employing in addition a transformation to polar coordinates this explains the following definition of a discrete $L^{2}$-norm for functions $u \in H^{2}\left(\mathbb{R}^{2}\right) \subset C\left(\mathbb{R}^{2}\right)$

$$
\begin{aligned}
& \|u\|_{\kappa \mu}^{2}=\frac{1}{\mu} \sum_{j=0}^{\kappa-1} \sum_{s=0}^{\mu-1} w_{j \kappa}\left|u\left(r_{j \kappa} \cos \vartheta_{s \mu}, r_{j \kappa} \sin \vartheta_{s \mu}\right)\right|^{2} \\
& \approx\|u\|_{L^{2}}^{2}=\int_{\mathbb{R}^{2}}|u(x, y)|^{2} \mathrm{~d}(x, y)=\int_{0}^{\infty} r|u(r \cos \vartheta, r \sin \vartheta)|^{2} \mathrm{~d} \vartheta \mathrm{d} r .
\end{aligned}
$$


The following result relates the discrete $L^{2}$-norm to the $L^{2}$-norm and Sobolev-seminorms; its proof relies on the Sobolev-type inequality provided by Lemma 5 and the above relations on the asymptotic distribution of the Gauß-Laguerre quadrature nodes and weights.

Lemma 6 For any $u \in H^{2}\left(\mathbb{R}^{2}\right)$ the bound

$$
\begin{aligned}
\|u\|_{\kappa \mu} & \leq \mathcal{C}\left(\|u\|_{L^{2}}+\left(\kappa^{-1 / 6}+\mu^{-1} \kappa^{\frac{1}{2}}\right)|u|_{H^{1}}+\left(\mu^{-1} \kappa^{\frac{1}{3}}+\kappa^{-\frac{1}{2}}\right)|u|_{H^{2}}\right) \\
& \leq \mathcal{C}\left(\|u\|_{L^{2}}+\mu^{-1 / 6}|u|_{H^{1}}+\mu^{-\frac{1}{2}}|u|_{H^{2}}\right)
\end{aligned}
$$

is valid, where in the second estimate the positive integers $\kappa, \mu \in \mathbb{N}_{\geq 1}$ are chosen proportional to each other.

Proof We first deduce a suitable estimate $(j=1, \ldots, \kappa-1, s=0, \ldots, \mu-1)$

$$
\frac{1}{\mu} w_{j \kappa}\left|u\left(r_{j \kappa} \cos \vartheta_{s \mu}, r_{j \kappa} \sin \vartheta_{s \mu}\right)\right|^{2} \leq \frac{1}{\mu} w_{j \kappa} \max _{(x, y) \in \bar{R}_{j \mu} \mu}|u(x, y)|^{2}
$$

where $R_{j s}^{\kappa \mu}$ denotes the curved rectangle

$$
R_{j s}^{\kappa \mu}=\left\{(x, y)=(r \cos \vartheta, r \sin \vartheta): r \in\left(r_{j-1, \kappa}, r_{j \kappa}\right), \vartheta \in\left(\vartheta_{s \mu}, \vartheta_{s+1, \mu}\right)\right\}
$$

the remaining contributions in (17) for $j=0, s=0, \ldots, \mu-1$ will be treated separately. We apply Lemma 5 and the above estimates (16), which in terms of the scaled Gauß-Laguerre quadrature nodes and weights yields the bounds

$$
\begin{gathered}
\mathcal{C}_{1} \kappa^{-1} \leq r_{j \kappa}^{2} \leq \mathcal{C}_{2} \kappa, \quad j=0,1, \ldots, \kappa-1, \\
\mathcal{C}_{3} \kappa^{-1} \leq \mathcal{C}_{4} w_{j \kappa} \leq \mathcal{C}_{5}\left(r_{j \kappa}^{2}-r_{j-1, \kappa}^{2}\right) \leq \mathcal{C}_{6} w_{j \kappa} \leq \mathcal{C}_{7} \kappa^{\frac{1}{3}}, \quad j=1,2, \ldots, \kappa-1, \\
w_{j \kappa} \frac{r_{j \kappa}^{2}-r_{j-1, \kappa}^{2}}{r_{j-1, \kappa}^{2}} \leq \mathcal{C} \frac{\varphi_{\kappa}\left(\varrho_{j \kappa}\right)}{\varrho_{j-1, \kappa}} \leq \mathcal{C} \frac{\varphi_{\kappa}\left(\varrho_{j-1, \kappa}\right)}{\varrho_{j-1, \kappa}} \leq \mathcal{C}_{8} \kappa^{-\frac{1}{3}}, \quad j=1,2, \ldots, \kappa-1,
\end{gathered}
$$

involving constants that are independent of $\kappa$; here, we applied the estimate $\varphi_{\kappa}\left(\varrho_{j \kappa}\right) \leq \mathcal{C} \varphi_{\kappa}\left(\varrho_{j-1, \kappa}\right)$ which follows from [15, Eq. (7.14), Thm 7.3 (c)] and (16f). It remains to estimate the constants arising in Lemma 5 with the help of the above relations

$$
\begin{aligned}
\frac{1}{\mu} w_{j \kappa} c_{0}^{2} & \leq \mathcal{C} \frac{1}{\left(\vartheta_{s+1, \mu}-\vartheta_{s \mu}\right) \mu} \frac{w_{j \kappa}}{r_{j \kappa}^{2}-r_{j-1, \kappa}^{2}} \leq \mathcal{C}, \\
\frac{1}{\mu} w_{j \kappa} c_{11}^{2} & \leq \mathcal{C} \frac{1}{\left(\vartheta_{s+1, \mu}-\vartheta_{s \mu}\right) \mu} w_{j \kappa} \frac{r_{j \kappa}^{2}-r_{j-1, \kappa}^{2}}{r_{j-1, \kappa}^{2}} \leq \mathcal{C} \kappa^{-\frac{1}{3}}, \\
\frac{1}{\mu} w_{j \kappa} c_{12}^{2} & \leq \mathcal{C} \frac{1}{\mu}\left(\vartheta_{s+1, \mu}-\vartheta_{s \mu}\right) w_{j \kappa} \frac{r_{j-1, \kappa}+r_{j \kappa}}{r_{j \kappa}-r_{j-1, \kappa}} \\
& \leq \mathcal{C} \mu^{-2}\left(r_{j, \kappa}^{2}-r_{j-1, \kappa}^{2}\right) \frac{r_{j-1, \kappa}+r_{j \kappa}}{r_{j \kappa}-r_{j-1, \kappa}}=\mathcal{C} \mu^{-2}\left(r_{j-1, \kappa}+r_{j \kappa}\right)^{2} \leq \mathcal{C} \mu^{-2} \kappa, \\
\frac{1}{\mu} w_{j \kappa} c_{13}^{2} & \leq \mathcal{C} \frac{1}{\mu}\left(\vartheta_{s+1, \mu}-\vartheta_{s \mu}\right) w_{j \kappa} \frac{r_{j \kappa}^{2}-r_{j-1, \kappa}^{2}}{r_{j-1, \kappa}^{2}} \leq \mathcal{C} \mu^{-2} \kappa^{-\frac{1}{3}}, \\
\frac{1}{\mu} w_{j \kappa} c_{2}^{2} & \leq \mathcal{C} \frac{1}{\mu}\left(\vartheta_{s+1, \mu}-\vartheta_{s \mu}\right) w_{j \kappa}\left(r_{j \kappa}^{2}-r_{j-1, \kappa}^{2}\right) \leq \mathcal{C} \mu^{-2} \kappa^{\frac{2}{3}} .
\end{aligned}
$$


Using these estimates together with the arithmetic-geometric mean inequality and applying the following relation for disjoint domains

$$
\|u\|_{L^{2}\left(\Omega_{1}\right)}^{2}+\|u\|_{L^{2}\left(\Omega_{2}\right)}^{2}=\int_{\Omega_{1}}|u|^{2}+\int_{\Omega_{2}}|u|^{2}=\int_{\Omega_{1} \cup \Omega_{2}}|u|^{2}=\|u\|_{L^{2}\left(\Omega_{1} \cup \Omega_{2}\right)}^{2},
$$

we obtain the estimate

$$
\begin{aligned}
& \frac{1}{\mu} \sum_{j=1}^{\kappa-1} \sum_{s=0}^{\mu-1} w_{j \kappa}\left|u\left(r_{j \kappa} \cos \vartheta_{s \mu}, r_{j \kappa} \sin \vartheta_{s \mu}\right)\right|^{2} \\
& \quad \leq \mathcal{C}\left(\|u\|_{L^{2}}^{2}+\left(\kappa^{-\frac{1}{3}}+\mu^{-2} \kappa\right)|u|_{H^{1}}^{2}+\mu^{-2} \kappa^{\frac{2}{3}}|u|_{H^{2}}^{2}\right)
\end{aligned}
$$

Similar arguments lead to the bound

$$
\frac{1}{\mu} w_{1 \kappa} \sum_{s=0}^{\mu-1}\left|u\left(r_{1 \kappa} \cos \vartheta_{s \mu}, r_{1 \kappa} \sin \vartheta_{s \mu}\right)\right|^{2} \leq w_{1 \kappa}\|u\|_{L^{\infty}} \leq \mathcal{C} \kappa^{-1}\|u\|_{H^{2}} .
$$

Altogether, the stated result follows.

4.6 Generalized-Laguerre-Fourier spectral interpolant

In the following, we introduce the generalized-Laguerre-Fourier spectral interpolant, obtained from the spectral representation by a restriction to finitely many basis functions and the application of the Gauß-Laguerre quadrature formula as well as the trapezoidal rule for the numerical approximation of the spectral coefficients. Moreover, we state an estimate for the generalizedLaguerre-Fourier spectral interpolant; together with Lemma 6 this fundamental result is needed in order to deduce a stability bound for the fully discrete evolution operator.

Notation. For the following, we fix positive integers $(K, M) \in \mathbb{N}_{\geq 1} \times \mathbb{N}_{\geq 2}$, where we assume that $M$ is an even number, and employ the abbreviations

$$
\begin{gathered}
\mathcal{M}_{K M}=\{0,1, \ldots, K-1\} \times\left\{-\frac{M}{2},-\frac{M}{2}+1, \ldots, \frac{M}{2}-1\right\} \subset \mathcal{M}=\mathbb{N} \times \mathbb{Z}, \\
\mathcal{K}_{K M}=\left\{0,1, \ldots, K+\frac{M}{2}-1\right\} \times\{0,1, \ldots, M-1\} \subset \mathbb{N} \times \mathbb{N} .
\end{gathered}
$$

For the zeros of the (standard) Laguerre polynomial of degree $K+\frac{M}{2}$, the associated scaled Gauß-Laguerre nodes and weights, the equidistant nodes associated with the trapezoidal rule, and the corresponding nodes in Cartesian coordinates, we write

$$
\begin{gathered}
\varrho_{j}=\varrho_{j \kappa}, \quad \omega_{j}=\omega_{j \kappa}, \quad r_{j}=\sqrt{\frac{\varrho_{j}}{\gamma}}, \quad w_{j}=\frac{\pi}{\gamma} \omega_{j} \mathrm{e}^{\varrho_{j}}, \quad \vartheta_{s}=\vartheta_{s M}, \\
x_{j s}=r_{j} \cos \vartheta_{s}, \quad y_{j s}=r_{j} \sin \vartheta_{s}, \quad(j, s) \in \mathcal{K}_{K M},
\end{gathered}
$$

for short, see also (15) as well as (17) with $\kappa=K+\frac{M}{2}$ and $\mu=M$. 
Orthogonal projection and spectral interpolant. For convenience, we recall the spectral representation with respect to the generalized-Laguerre-Fourier basis functions

$$
u=\sum_{(k, m) \in \mathcal{M}} c_{k m}(u) \mathcal{L}_{k m}^{\gamma}, \quad u \in L^{2}\left(\mathbb{R}^{2}\right)
$$

involving the complex coefficients

$$
\begin{aligned}
c_{k m}(u) & =\left(\mathcal{L}_{k m}^{\gamma} \mid u\right)_{L^{2}}=\int_{\mathbb{R}^{2}} \overline{\mathcal{L}_{k m}^{\gamma}(x, y)} u(x, y) \mathrm{d}(x, y) \\
& =\int_{0}^{\infty} \int_{0}^{2 \pi} r \widetilde{L}_{k,|m|}^{\gamma}(r) \mathrm{e}^{-\mathrm{i} m \vartheta} u(r \cos \vartheta, r \sin \vartheta) \mathrm{d} \vartheta \mathrm{d} r
\end{aligned}
$$

see (10) and (11). The orthogonal projection onto the subspace

$$
X_{K M}=\operatorname{span}\left\{\mathcal{L}_{k m}^{\gamma}:(k, m) \in \mathcal{M}_{K M}\right\}
$$

is defined by

$$
\mathcal{P}_{K M}(u)=\sum_{(k, m) \in \mathcal{M}_{K M}} c_{k m}(u) \mathcal{L}_{k m}^{\gamma}
$$

In accordance with (17), we define the spectral interpolant by

$$
\begin{gathered}
\mathcal{Q}_{K M} u=\sum_{(k, m) \in \mathcal{M}_{K M}} \widetilde{c}_{k m}(u) \mathcal{L}_{k m}^{\gamma}, \\
\widetilde{c}_{k m}(u)=\frac{1}{M} \sum_{(j, s) \in \mathcal{K}_{K M}} w_{j} \overline{\mathcal{L}_{k m}^{\gamma}\left(x_{j s}, y_{j s}\right)} u\left(x_{j s}, y_{j s}\right),
\end{gathered}
$$

that is, approximations $\widetilde{c}_{k m}(u) \approx c_{k m}(u)$ are obtained by a representation of the integral in polar coordinates, the substitution $\varrho=\gamma r^{2}$, and applications of the Gauß-Laguerre quadrature formula as well as the trapezoidal rule; evidently, the spectral interpolant is well-defined for any function $u \in C\left(\mathbb{R}^{2}\right)$. We point out that the interpolation property at the quadrature nodes only holds approximately, i.e.,

$$
u\left(x_{j s}, y_{j s}\right) \approx\left(\mathcal{Q}_{K M} u\right)\left(x_{j s}, y_{j s}\right), \quad(j, s) \in \mathcal{K}_{K M},
$$

since the number of interpolation points $\# \mathcal{K}_{K M}=\left(K+\frac{M}{2}\right) M$ exceeds the number of basis functions $\# \mathcal{M}_{K M}=K M$. However, the choice $\kappa=K+\frac{M}{2}$ and not merely $\kappa=K$ is needed in order to ensure exact quadrature, which then also ensures the validity of a basic discrete orthogonality relation. 
Basic relations for spectral interpolants. Fundamental relations and a first estimate for the generalized-Laguerre-Fourier spectral interpolant are provided by the following result; we recall the definition of the discrete $L^{2}$-norm, see (17).

Lemma 7 (i) The spectral basis functions $\left(\mathcal{L}_{k m}\right)_{(k, m) \in \mathcal{M}_{K M}}$ satisfy the discrete orthogonality relation

$$
\frac{1}{M} \sum_{(j, s) \in \mathcal{K}_{K M}} w_{j} \overline{\mathcal{L}_{k m}^{\gamma}\left(x_{j s}, y_{j s}\right)} \mathcal{L}_{k^{\prime} m^{\prime}}^{\gamma}\left(x_{j s}, y_{j s}\right)=\delta_{k k^{\prime}} \delta_{m m^{\prime}}
$$

for all $(k, m),\left(k^{\prime}, m^{\prime}\right) \in \mathcal{M}_{K M}$.

(ii) For any $u \in L^{2}\left(\mathbb{R}^{2}\right)$ the identity

$$
\mathcal{Q}_{K M} \mathcal{P}_{K M} u=\mathcal{P}_{K M} u
$$

holds.

(iii) For any $u \in C\left(\mathbb{R}^{2}\right)$ the bound

$$
\left\|\mathcal{Q}_{K M} u\right\|_{L^{2}}=\left\|\mathcal{Q}_{K M} u\right\|_{K+\frac{M}{2}, M} \leq\|u\|_{K+\frac{M}{2}, M}
$$

is valid.

Proof (i) By definition (10) and the discrete orthogonality relation for the Fourier spectral method $\left(m, m^{\prime}=-\frac{M}{2}, \ldots, \frac{M}{2}-1\right)$

$$
\frac{1}{M} \sum_{s=0}^{M-1} \mathrm{e}^{-\mathrm{i} m \vartheta_{s}} \mathrm{e}^{\mathrm{i} m^{\prime} \vartheta_{s}}=\delta_{m m^{\prime}},
$$

easily shown with the help of the geometric series, we obtain the identity $\left((k, m),\left(k^{\prime}, m^{\prime}\right) \in \mathcal{M}_{K M}\right)$

$$
\begin{aligned}
& \frac{1}{M} \sum_{(j, s) \in \mathcal{K}_{K M}} w_{j} \overline{\mathcal{L}_{k m}^{\gamma}\left(x_{j s}, y_{j s}\right)} \mathcal{L}_{k^{\prime} m^{\prime}}^{\gamma}\left(x_{j s}, y_{j s}\right) \\
& =\left(\frac{1}{M} \sum_{s=0}^{M-1} \mathrm{e}^{\mathrm{i}\left(m^{\prime}-m\right) \vartheta_{s}}\right)\left(\sum_{j=0}^{K+\frac{M}{2}-1} w_{j} \widetilde{L}_{k,|m|}^{\gamma}\left(r_{j}\right) \widetilde{L}_{k^{\prime},\left|m^{\prime}\right|}^{\gamma}\left(r_{j}\right)\right) \\
& =\delta_{m m^{\prime}} \sum_{j=0}^{K+\frac{M}{2}-1} w_{j} \widetilde{L}_{k,|m|}^{\gamma}\left(r_{j}\right) \widetilde{L}_{k^{\prime},|m|}^{\gamma}\left(r_{j}\right),
\end{aligned}
$$

see also Section 4.1. Recalling the definition (9) of the scaled generalizedLaguerre functions, the exactness of the Gauß-Laguerre quadrature formula for polynomials of degree at most $2 K+M-1$, especially fulfilled for the polynomial $\varrho \mapsto \varrho^{m} L_{k}^{m}(\varrho) L_{k^{\prime}}^{m}(\varrho)$ of degree at most $2 K+\frac{M}{2}-2$, together with 
the orthogonality relation for the generalized-Laguerre polynomials further imply $\left(k, k^{\prime}=0,1, \ldots, K-1, m=0,1, \ldots, \frac{M}{2}\right)$

$$
\begin{aligned}
& \sum_{j=0}^{K+\frac{M}{2}-1} w_{j} \widetilde{L}_{k m}^{\gamma}\left(r_{j}\right) \widetilde{L}_{k^{\prime} m}^{\gamma}\left(r_{j}\right)=\frac{\pi}{\gamma} \sum_{j=0}^{K+\frac{M}{2}-1} \omega_{j} \mathrm{e}^{\varrho_{j}} \widetilde{L}_{k m}^{\gamma}\left(\sqrt{\frac{\varrho_{j}}{\gamma}}\right) \widetilde{L}_{k^{\prime} m}^{\gamma}\left(\sqrt{\frac{\varrho_{j}}{\gamma}}\right) \\
& \quad=\frac{1}{\sqrt{C_{k}^{m} C_{k^{\prime}}^{m}}} \sum_{j=0}^{K+\frac{M}{2}-1} \omega_{j} \varrho_{j}^{m} L_{k}^{m}\left(\varrho_{j}\right) L_{k^{\prime}}^{m}\left(\varrho_{j}\right) \\
& \quad=\frac{1}{\sqrt{C_{k}^{m} C_{k^{\prime}}^{m}}} \int_{0}^{\infty} r^{m} \mathrm{e}^{-r} L_{k}^{m}(r) L_{k^{\prime}}^{m}(r) \mathrm{d} r \\
& =\delta_{k k^{\prime}},
\end{aligned}
$$

which proves the first statement.

(ii) The aim is to show that for a function of the form

$$
u=\sum_{(k, m) \in \mathcal{M}_{K M}} c_{k m}(u) \mathcal{L}_{k m}^{\gamma}
$$

the relation $\widetilde{c}_{k m}(u)=c_{k m}(u)$ holds for all $(k, m) \in \mathcal{M}$, see (20). Due to linearity it is sufficient to consider $u=\mathcal{L}_{k^{\prime} m^{\prime}}^{\gamma}$ for $\left(k^{\prime}, m^{\prime}\right) \in \mathcal{M}_{K M}$, where the assertion follows at once by the orthogonality relation for the basis functions and its discrete analogue $\left((k, m),\left(k^{\prime}, m^{\prime}\right) \in \mathcal{M}_{K M}\right)$

$$
\begin{aligned}
\widetilde{c}_{k m}\left(\mathcal{L}_{k^{\prime} m^{\prime}}^{\gamma}\right) & =\frac{1}{M} \sum_{(j, s) \in \mathcal{K}_{K M}} w_{j} \overline{\mathcal{L}_{k m}^{\gamma}\left(x_{j s}, y_{j s}\right)} \mathcal{L}_{k^{\prime} m^{\prime}}^{\gamma}\left(x_{j s}, y_{j s}\right) \\
& =\delta_{k k^{\prime}} \delta_{m m^{\prime}}=\left(\mathcal{L}_{k m}^{\gamma} \mid \mathcal{L}_{k^{\prime} m^{\prime}}^{\gamma}\right)_{L^{2}}=c_{k m}\left(\mathcal{L}_{k m}^{\gamma}\right) .
\end{aligned}
$$

(iii) For the following considerations it is convenient to employ compact matrix notations. For a given function $u \in C\left(\mathbb{R}^{2}\right)$ we collect the function values at the interpolation points in a column vector, with indices $(j, s) \in \mathcal{K}_{K M}$ occurring in the order $(0,0), \ldots,\left(K+\frac{M}{2}-1,0\right),(0,1), \ldots,\left(K+\frac{M}{2}-1, M-1\right)$, and in a similar manner we collect the corresponding spectral coefficients in a column vector, with indices $(k, m) \in \mathcal{M}_{K M}$ occurring in the order $(0,0), \ldots,(K-1,0),(0,1), \ldots,(K-1, M-1)$

$$
u=\left(u\left(x_{j s}, y_{j s}\right)\right) \in \mathbb{R}^{\left(K+\frac{M}{2}\right) M}, \quad \hat{u}=\left(\widetilde{c}_{k m}(u)\right) \in \mathbb{R}^{K M} .
$$

In addition, we introduce a diagonal matrix comprising the quadrature weights as well as a matrix comprising the values of the basis functions at the quadrature nodes

$$
\begin{aligned}
W & =\frac{1}{M} \operatorname{diag}\left(w_{j}\right) \in \mathbb{R}^{\left(K+\frac{M}{2}\right) M \times\left(K+\frac{M}{2}\right) M}, \\
L & =\left(\mathcal{L}_{k m}^{\gamma}\left(x_{j s}, y_{j s}\right)\right) \in \mathbb{R}^{K M \times\left(K+\frac{M}{2}\right) M},
\end{aligned}
$$

where the weights $\left(w_{j}\right)_{j=0}^{K+\frac{M}{2}-1}$ occur $M$ times, with indices $(j, s)$ indexing columns and indices $(k, m)$ indexing rows in the same orders as in the vectors $u$ 
and $\hat{u}$, respectively. The spectral transformation and the discrete orthogonality relation are then given by

$$
\hat{u}=L^{\dagger} W u, \quad L^{\dagger} W L=I,
$$

where $L^{\dagger}$ denotes the conjugate transpose of the matrix $L$. The evaluation of the spectral interpolant at the interpolation points corresponds to the mapping $\hat{\mathbf{u}} \mapsto L \hat{\mathbf{u}}$, and the vector comprising the values of the spectral interpolant at the interpolation points is given by

$$
\left(\mathcal{Q}_{K M} u\left(x_{j s}, y_{j s}\right)\right)=\mathcal{Q} u=L \hat{u}=L L^{\dagger} W u \in \mathbb{R}^{\left(K+\frac{M}{2}\right) M} .
$$

Moreover, the discrete $L^{2}$-norm defined in (17) equals

$$
\|u\|_{K+\frac{M}{2}, M}^{2}=u^{\dagger} W u \text {. }
$$

As a consequence, by Parseval's identity and the discrete orthogonality relation the identity

$$
\begin{aligned}
\left\|\mathcal{Q}_{K M} u\right\|_{L^{2}}^{2} & =\sum_{(k, m) \in \mathcal{M}_{K M}}\left|\widetilde{c}_{k m}(u)\right|^{2}=\hat{u}^{\dagger} \hat{u}=\left(L^{\dagger} W u\right)^{\dagger}\left(L^{\dagger} W u\right) \\
& =u^{\dagger} W L L^{\dagger} W u=u^{\dagger} W L L^{\dagger} W L L^{\dagger} W u \\
& =\left(L L^{\dagger} W u\right)^{\dagger} W\left(L L^{\dagger} W u\right)=(\mathcal{Q} u)^{\dagger} W(\mathcal{Q} u)=\|\mathcal{Q} u\|_{K+\frac{M}{2}, M}^{2}
\end{aligned}
$$

follows. As the orthogonality relation $L^{\dagger} W L=I$ implies

$$
W L L^{\dagger} W\left(I-L L^{\dagger} W\right)=W L L^{\dagger} W-W L L^{\dagger} W L L^{\dagger} W=0,
$$

we finally obtain the estimate

$$
\begin{aligned}
\|u\|_{K+\frac{M}{2}, M}^{2}= & \left\|\mathcal{Q}_{K M} u+\left(I-\mathcal{Q}_{K M}\right) u\right\|_{K+\frac{M}{2}, M}^{2} \\
= & (\mathcal{Q} u+(I-\mathcal{Q}) u)^{\dagger} W(\mathcal{Q} u+(I-\mathcal{Q}) u) \\
= & u^{\dagger}\left(L L^{\dagger} W+\left(I-L L^{\dagger} W\right)\right)^{\dagger} W\left(L L^{\dagger} W+\left(I-L L^{\dagger} W\right)\right) u \\
= & \|\mathcal{Q} u\|_{K+\frac{M}{2}, M}^{2}+\|(I-\mathcal{Q}) u\|_{K+\frac{M}{2}, M}^{2} \\
& \quad+2 \Re\left(u^{\dagger} W L L^{\dagger} W\left(I-L L^{\dagger} W\right) u\right) \\
\geq & \left\|\mathcal{Q}_{K M} u\right\|_{K+\frac{M}{2}, M}^{2}=\left\|\mathcal{Q}_{K M} u\right\|_{L^{2}}^{2}
\end{aligned}
$$

which proves the assertion. 
Estimates for spectral interpolants in fractional power spaces. The following result provides estimates for the generalized-Laguerre-Fourier spectral interpolant in fractional power spaces associated with the dominant linear part. We recall the definition of the fractional power spaces and note that the spectral interpolant is well defined for any function $u \in X_{\alpha}$ with $\alpha \in \mathbb{N}_{\geq 1}$, see Section 4.2 and Lemma 3. We denote by

$$
\lambda_{\max }=\max _{(k, m) \in \mathcal{M}_{K M}} \lambda_{k m} \leq \mathcal{C}(K+M)
$$

the maximal eigenvalue in the index set $\mathcal{M}_{K M}$ and further set

$$
\|u\|_{L^{\infty}\left(\mathcal{K}_{K M}\right)}=\max \left\{\left|u\left(x_{j s}, y_{j s}\right)\right|:(j, s) \in \mathcal{K}_{K M}\right\},
$$

see also $(11 d)$.

Lemma 8 Let $(K, M) \in \mathbb{N}_{\geq 1} \times \mathbb{N}_{\geq 1}$ with $M$ an even number and $\alpha \in \mathbb{N}_{\geq 1}$.

(i) For all $u, v \in X_{\alpha}$ the relations

$$
\begin{gathered}
\left\|\mathcal{Q}_{K M} u\right\|_{X_{\alpha}} \leq \lambda_{\max }^{\alpha}\left\|\mathcal{Q}_{K M} u\right\|_{X_{0}}, \\
\left\|\mathcal{Q}_{K M}(u v)\right\|_{X_{0}} \leq\|u\|_{L^{\infty}\left(\mathcal{K}_{K M}\right)}\|v\|_{K+\frac{M}{2}, M} \leq \mathcal{C}\|u\|_{X_{\alpha}}\|v\|_{K+\frac{M}{2}, M},
\end{gathered}
$$

are valid.

(ii) Provided that the positive integers $K$ and $M$ are proportional, for any $u \in X_{\alpha}$ and for $0 \leq \zeta \leq \alpha$ the estimates

$$
\begin{aligned}
\left\|\mathcal{Q}_{K M} u\right\|_{X_{0}} & \leq\|u\|_{K+\frac{M}{2}, M} \\
& \leq \mathcal{C}\left(\|u\|_{X_{0}}+M^{-1 / 6}\|u\|_{X_{\frac{1}{2}}}+M^{-\frac{1}{2}}\|u\|_{X_{1}}\right) \leq \mathcal{C}\|u\|_{X_{\alpha}}, \\
\left\|\left(\mathcal{Q}_{K M}-I\right) u\right\|_{X_{\zeta}} & \leq \mathcal{C} \lambda_{\max }^{-(\alpha-\zeta)}\left(1+\lambda_{\max }^{\frac{1}{2}} M^{-1 / 6}+\lambda_{\max } M^{-\frac{1}{2}}\right)\|u\|_{X_{\alpha}} \\
& \leq \mathcal{C} M^{-\left(\alpha-\zeta-\frac{1}{2}\right)}\|u\|_{X_{\alpha}},
\end{aligned}
$$

are valid.

Proof We recall assumption (4) on the angular momentum rotation speed.

(i) The first statement follows immediately from the relation

$$
\left\|\mathcal{Q}_{K M} u\right\|_{X_{\alpha}}=\left\|\sum_{(k, m) \in \mathcal{M}_{K M}} \widetilde{c}_{k m}(u) \lambda_{k m}^{\alpha} \mathcal{L}_{k m}^{\gamma}\right\|_{X_{0}} \leq \lambda_{\max }^{\alpha}\left\|\mathcal{Q}_{K M} u\right\|_{X_{0}}
$$

and by Lemma 3 we obtain

$$
\begin{aligned}
\left\|\mathcal{Q}_{K M}(u v)\right\|_{X_{0}}^{2} & =\left\|\mathcal{Q}_{K M}(u v)\right\|_{K+\frac{M}{2}, M}^{2} \\
& =\frac{1}{M} \sum_{(j, s) \in \mathcal{K}_{K, M}} w_{j}\left|u\left(x_{j s}, y_{j s}\right)\right|^{2}\left|v\left(x_{j s}, y_{j s}\right)\right|^{2} \\
& \leq\|u\|_{L^{\infty}\left(\mathcal{K}_{K M}\right)}^{2}\|v\|_{K+\frac{M}{2}}^{2} \leq \mathcal{C}\|u\|_{X_{\alpha}}^{2}\|v\|_{K+\frac{M}{2}, M}^{2}
\end{aligned}
$$


(ii) We apply Lemma 3 as well as Lemma 6 . For $u \in X_{\alpha}$ with $\alpha \in \mathbb{N}_{\geq 1}$ and for $0 \leq \zeta \leq \alpha$, due to

$$
A^{\zeta}\left(\mathcal{P}_{K M}-I\right) u=-\sum_{(k, m) \in \mathcal{M} \backslash \mathcal{M}_{K M}} c_{k m}(u) \lambda_{k m}^{\zeta} \mathcal{L}_{k m}^{\gamma},
$$

we obtain the estimate

$$
\begin{aligned}
\left\|A^{\zeta}\left(\mathcal{P}_{K M}-I\right) u\right\|_{X_{0}}^{2} & =\sum_{(k, m) \in \mathcal{M} \backslash \mathcal{M}_{K M}}\left|c_{k m}(u)\right|^{2} \lambda_{k m}^{2 \zeta} \\
& \leq \lambda_{\max }^{-2(\alpha-\zeta)} \sum_{(k, m) \in \mathcal{M} \backslash \mathcal{M}_{K M}}\left|c_{k m}(u)\right|^{2} \lambda_{k m}^{2 \alpha} \\
& \leq \lambda_{\max }^{-2(\alpha-\zeta)} \sum_{(k, m) \in \mathcal{M}}\left|c_{k m}(u)\right|^{2} \lambda_{k m}^{2 \alpha}=\lambda_{\max }^{-2(\alpha-\zeta)}\|u\|_{X_{\alpha}}^{2} .
\end{aligned}
$$

Applying the identity

$\mathcal{Q}_{K M}-I=\left(\mathcal{Q}_{K M}-\mathcal{P}_{K M}\right)+\left(\mathcal{P}_{K M}-I\right)=\mathcal{Q}_{K M}\left(I-\mathcal{P}_{K M}\right)+\left(\mathcal{P}_{K M}-I\right)$,

the assertion follows from

$$
\begin{aligned}
\left\|\left(\mathcal{Q}_{K M}-I\right) u\right\|_{X_{\zeta}} \leq & \left\|\mathcal{Q}_{K M}\left(I-\mathcal{P}_{K M}\right) u\right\|_{X_{\zeta}}+\left\|\left(\mathcal{P}_{K M}-I\right) u\right\|_{X_{\zeta}} \\
\leq & \lambda_{\max }^{\zeta}\left\|\mathcal{Q}_{K M}\left(I-\mathcal{P}_{K M}\right) u\right\|_{X_{0}}+\lambda_{\max }^{-(\alpha-\zeta)}\|u\|_{X_{\alpha}} \\
\leq & \mathcal{C} \lambda_{\max }^{\zeta}\left(\left\|\left(I-\mathcal{P}_{K M}\right) u\right\|_{X_{0}}+M^{-1 / 6}\left\|\left(I-\mathcal{P}_{K M}\right) u\right\|_{X_{\frac{1}{2}}}\right. \\
& \left.+M^{-\frac{1}{2}}\left\|\left(I-\mathcal{P}_{K M}\right) u\right\|_{X_{1}}\right)+\lambda_{\max }^{-(\alpha-\zeta)}\|u\|_{X_{\alpha}} \\
\leq & \mathcal{C} \lambda_{\max }^{-(\alpha-\zeta)}\left(1+\lambda_{\max }^{\frac{1}{2}} M^{-1 / 6}+\lambda_{\max } M^{-\frac{1}{2}}\right)\|u\|_{X_{\alpha}} ;
\end{aligned}
$$

note that for integers $K$ proportional to $M$ we may use that $\lambda_{\max } \leq \mathcal{C} M$. $\diamond$

\section{Convergence analysis}

This section is devoted to the derivation of a convergence result for full discretizations of time-dependent Gross-Pitaevskii equations with additional rotation term by time-splitting generalized-Fourier-Laguerre-Hermite pseudospectral methods, see Theorem 1. Our approach in the lines of $[10,17,23]$ in particular utilizes the stability and error analysis for semi-discretizations in time given therein. In order to keep the manuscript at a reasonable length, we focus on the second-order Strang splitting method and do not specify the local error expansion found in [17]; based on the general approach [23] and the auxiliary results provided in Section 4 the analysis extends to higher-order splitting methods. We meanwhile restrict ourselves to the case of two space dimensions; the extension to the three-dimensional Gross-Pitaevskii equation is indicated in Section 5.3. For simplicity, we henceforth assume that the space 
discretization parameters are proportional; thus, it suffices to indicate the dependence of the spectral interpolation operator and the numerical solution on the even integer $M \in \mathbb{N}_{\geq 2}$. The consideration of a constant time stepsize $\Delta t>0$ facilitates a minor simplification in notation.

Space and time discretization (Strang). For convenience, we recall the recurrence relations

$$
\begin{gathered}
u_{M}^{n+1}=\mathcal{F}_{M}(\Delta t) u_{M}^{n}=\mathrm{e}^{-\frac{1}{2} \mathrm{i} \Delta t A} \mathcal{Q}_{M} \mathrm{e}^{-\mathrm{i} \Delta t B\left[U_{M}^{n}\right]} U_{M}^{n}, \\
U_{M}^{n}=\mathrm{e}^{-\frac{1}{2} \mathrm{i} \Delta t A} \mathcal{Q}_{M} u_{M}^{n}, \\
u^{n+1}=\mathcal{S}(\Delta t) u^{n}=\mathrm{e}^{-\frac{1}{2} \mathrm{i} \Delta t A} \mathrm{e}^{-\mathrm{i} \Delta t B\left[U^{n}\right]} U^{n}, \quad U^{n}=\mathrm{e}^{-\frac{1}{2} \mathrm{i} \Delta t A} u^{n},
\end{gathered}
$$

for the fully discrete solution $\left(u_{M}^{n}\right)_{n=0}^{N}$ to (2) and its time-discrete analogue $\left(u^{n}\right)_{n=0}^{N}$, employing formally linear notations, see also (5) and Section 4.6 for the definition of the spectral interpolation operator.

\subsection{Semi-discretization in time}

In order to establish a convergence estimate for the Strang time-splitting method applied to the time-dependent two-dimensional Gross-Pitaevskii equation with rotation term, we pursue the standard approach of combining stability bounds and local error estimates. We note that our approach following [23] is general and permits to cover different spectral methods; the particular choice of the specific spectral method enters in the definition of the operators $A, B$ and the auxiliary results deduced in Section 4 .

\subsubsection{Stability}

Estimates for the evolution operator associated with $B$. Bounds for the action of the operator $B$ and the associated evolution operator in fractional power spaces are provided by the following result. By means of the auxiliary results deduced in Section 4.3 the corresponding estimates given in [23] carry over literally, see also $[10,17]$.

Lemma 9 Let $\alpha \in \mathbb{N}_{\geq 1}$ and set $\zeta=0$ or $\zeta=\alpha$, respectively. Then for $u \in X_{\alpha}$ and $v \in X_{\zeta}$ the bounds

$$
\begin{gathered}
\|B[u] v\|_{X_{\zeta}} \leq \mathcal{C}\left(\|V\|_{X_{\alpha}}+|\beta|\|u\|_{X_{\alpha}}^{2}\right)\|v\|_{X_{\zeta}}, \\
\left\|\mathrm{e}^{-\mathrm{i} t B[u]} v\right\|_{X_{\zeta}} \leq \mathrm{e}^{\mathcal{C}\left(\|V\|_{X_{\alpha}}+|\beta|\|u\|_{X_{\alpha}}^{2}\right) t}\|v\|_{X_{\zeta}}
\end{gathered}
$$

are valid. Furthermore, for $u, v, w \in X_{\alpha}$ the relation

$$
\|(B[u]-B[v]) w\|_{X_{\zeta}} \leq \mathcal{C}|\beta|\left(\|u\|_{X_{\alpha}}+\|v\|_{X_{\alpha}}\right)\|w\|_{X_{\alpha}}\|u-v\|_{X_{\zeta}}
$$

holds. 
Stability bound for the Strang semi-discretization in time. Arguments given in $[10,17,23]$ lead to stability bounds for time-spitting methods; we note that the evolution operator associated with $A$ is unitary on any fractional power space $\left(u \in X_{\alpha}, \alpha \in \mathbb{R}, t \in \mathbb{R}\right)$

$$
\left\|\mathrm{e}^{-\mathrm{i} t A} v\right\|_{X_{\alpha}}=\|u\|_{X_{\alpha}} .
$$

Lemma 10 For any $u, v \in X_{\alpha}$ with $\alpha \in \mathbb{N}_{\geq 1}$ and for $\zeta=0$ or $\zeta=\alpha$, respectively, the estimate

$$
\|\mathcal{S}(t) u-\mathcal{S}(t) v\|_{X_{\zeta}} \leq \mathrm{e}^{\mathcal{C}\left(\mathcal{C}_{V}+\mathcal{C}_{\alpha}^{2}|\beta|\right) t}\|u-v\|_{X_{\zeta}},
$$

holds, where $\mathcal{C}_{V}>0$ depends on an upper bound for $\|V\|_{X_{\alpha}}$ and $\mathcal{C}_{\alpha}>0$ depends on an upper bound for $\|u\|_{X_{\alpha}}$ as well as $\|v\|_{X_{\alpha}}$.

\subsubsection{Local error}

Commutator bounds. Essential ingredients in the derivation of local error estimates for time-splitting methods are bounds for iterated Lie-commutators. The following result provides estimates for the first and second iterated Liecommutators needed in connection with the second-order Strang splitting method. We note that in the proof the iterated commutators are expressed in terms of the linear operator $A$ as well as the potential $V$ and that the specific form of $A$ is not exploited; in the case of a nonlinear operator $B$ defining the problem this simplification is useful, however, in the linear case, in order to obtain bounds which are optimal with respect to the required regularity properties of $u$, the cancellation of terms has to be taken into account. Following [23] an analogous result for higher iterated Lie commutators arising in the local error analysis of higher-order time-splitting methods applied to (1) may be obtained. In the following, we set

$$
\widehat{A}(u)=-\mathrm{i} A u, \quad \widehat{B}(u)=-\mathrm{i} B[u] u=-\mathrm{i}\left(V+\beta|u|^{2}\right) u .
$$

Lemma 11 For every $u \in X_{\alpha+1}$ with $\alpha \in \mathbb{N}_{\geq 1}$ the bounds

$$
\begin{gathered}
\|[\widehat{A}, \widehat{B}](u)\|_{X_{\alpha}} \leq \mathcal{C}\left(|\beta|\|u\|_{X_{\alpha+1}}^{3}+\|V\|_{X_{\alpha+1}}\|u\|_{X_{\alpha+1}}\right), \\
\|[\widehat{A},[\widehat{A}, \widehat{B}]](u)\|_{X_{0}} \leq \mathcal{C}\left(|\beta|\|u\|_{X_{\alpha+1}}^{3}+\|V\|_{X_{\alpha+1}}\|u\|_{X_{\alpha+1}}\right),
\end{gathered}
$$

are valid.

Proof The Fréchet derivatives of $\widehat{A}$ and $\widehat{B}$ are given by

$$
\widehat{A}^{\prime}(u) v=-\mathrm{i} A v, \quad \widehat{B}^{\prime}(u) v=-\mathrm{i}\left(V v+2 \beta|u|^{2} v+\beta u^{2} v\right),
$$

respectively, so that

$$
\begin{aligned}
{[\widehat{A}, \widehat{B}](u) } & =\widehat{A}^{\prime}(u) \widehat{B}(u)-\widehat{B}^{\prime}(u) \widehat{A}(u) \\
& =-A\left(V u+\beta|u|^{2} u\right)+V A u+2 \beta|u|^{2} A u-\beta u^{2} \overline{A u} \\
& =-[A, V] u-\beta\left(A\left(|u|^{2} u\right)-2|u|^{2} A u+u^{2} \overline{A u}\right) .
\end{aligned}
$$


By the definition of the norm in the fractional power space $X_{\alpha}$ and Lemma 3 the bounds

$$
\begin{gathered}
\left\|A\left(|u|^{2} u\right)-2|u|^{2} A u+u^{2} \overline{A u}\right\|_{X_{\alpha}} \leq \mathcal{C}\|u\|_{X_{\alpha+1}}^{3}, \\
\|[A, V] u\|_{X_{\alpha}} \leq \mathcal{C}\|V\|_{X_{\alpha+1}}\|u\|_{X_{\alpha+1}}
\end{gathered}
$$

follow, which proves the first commutator bound. A straightforward estimation of the first term in

$$
[\widehat{A},[\widehat{A}, \widehat{B}]](u)=\widehat{A}^{\prime}(u)([\widehat{A}, \widehat{B}](u))-[\widehat{A}, \widehat{B}]^{\prime}(u)(\widehat{A} u),
$$

using that $\alpha \in \mathbb{N}_{\geq 1}$ by assumption, yields

$$
\left\|\widehat{A}^{\prime}(u)([\widehat{A}, \widehat{B}](u))\right\|_{X_{0}}=\|[\widehat{A}, \widehat{B}](u)\|_{X_{1}} \leq\|[\widehat{A}, \widehat{B}](u)\|_{X_{\alpha}} .
$$

The Fréchet derivative of the second term equals

$$
\begin{aligned}
{[\widehat{A}, \widehat{B}]^{\prime}(u) v=- } & {[A, V] v-\beta\left(A\left(2|u|^{2} v+u^{2} \bar{v}\right)-2 u \bar{v} A u-2 \bar{u} v A u\right.} \\
& \left.-2|u|^{2} A v+u^{2} \overline{A v}+2 u v \overline{A u}\right)
\end{aligned}
$$

which further implies

$$
\begin{aligned}
{[\widehat{A}, \widehat{B}]^{\prime}(u)(\widehat{A} u)=\mathrm{i}[A, V] A u+\mathrm{i} \beta\left(A\left(2|u|^{2} A u-u^{2} \overline{A u}\right)+2 u|A u|^{2}\right.} \\
\left.-2 \bar{u}(A u)^{2}-2|u|^{2} A^{2} u-u^{2} \overline{A^{2} u}+2 u|A u|^{2}\right) .
\end{aligned}
$$

An application of Lemma 3, using that

$$
\|u\|_{X_{2}}^{3} \leq \mathcal{C}\|u\|_{X_{\alpha+1}}^{3}, \quad\|V\|_{X_{2}}\|u\|_{X_{2}} \leq \mathcal{C}\|V\|_{X_{\alpha+1}}\|u\|_{X_{\alpha+1}},
$$

yields the statement.

Local error estimate. By means of a suitable local error expansion and the Liecommutator bounds provided by Lemma 11, it is straightforward to obtain the following local error estimate for the special case of a second-order splitting method; as the specification of the local error expansion deduced in [17] for the Strang splitting method and the generalization to high-order splitting methods given in $[14,23]$ utilizes the formal calculus of Lie-derivatives, we do not recapitulate the local error expansion in this work and refer to the literature for details of the proof.

Lemma 12 Consider an exponential operator splitting method of nonstiff order $p \in \mathbb{N}_{\geq 1}$ for the time integration of the nonlinear evolution equation (1)(2). For some initial value $u^{0} \in X_{p}$ let $u(\Delta t)$ denote the exact solution at time $t=\Delta t$. Then the local error estimates

$$
\left\|\mathcal{S}(\Delta t) u^{0}-u(\Delta t)\right\|_{X_{1}} \leq \mathcal{C}(\Delta t)^{p}, \quad\left\|\mathcal{S}(\Delta t) u^{0}-u(\Delta t)\right\|_{X_{0}} \leq \mathcal{C}(\Delta t)^{p+1}
$$

are valid with constants depending in particular on upper bounds for $\left\|u^{0}\right\|_{X_{p}}$ and $\|V\|_{X_{p}}$. 


\subsubsection{Global error}

Global error estimate. A standard approach based on the telescopic identity

$$
u^{n}-u\left(t_{n}\right)=\sum_{j=0}^{n-1} \prod_{\ell=j+1}^{n-1} \mathcal{S}(\Delta t)\left(\mathcal{S}(\Delta t) u\left(t_{j}\right)-u\left(t_{j}+\Delta t\right)\right)
$$

again in formally linear notation, yields an estimate for the global error in terms of stability bounds and local error estimates as provided by Lemmas 10 and 12 ; for simplicity, we here assume that the starting value $u^{0}$ coincides with the exact initial value $u(0)$. We omit a detailed proof and refer to [17] for the case of the second-order Strang splitting method. The generalization to high-order splitting methods is given in [23]; the error analysis shows that the nonstiff order of convergence is retained under suitable regularity requirements on the exact solution.

Theorem 2 Consider a variable stepsize exponential operator splitting method of nonstiff order $p \in \mathbb{N}_{>1}$ for the time discretization of the timedependent Gross-Pitaevskii equation (1)-(2). Under the assumption that the potential $V$ and the values of the exact solution remain bounded in the fractional power space $X_{p}$, the global error estimate

$$
\left\|u^{n}-u\left(t_{n}\right)\right\|_{X_{0}} \leq \mathcal{C}\left(\left\|u^{0}-u(0)\right\|_{X_{0}}+(\Delta t)^{p}\right), \quad 0 \leq t_{n} \leq t_{N} \leq T,
$$

is valid, where $\Delta t=\max \left\{\tau_{n}: n=0,1, \ldots, N-1\right\}$ denotes the maximal time increment; the arising constant $\mathcal{C}>0$ in particular depends on upper bounds for $\|V\|_{X_{p}}$ and $\max \left\{\|u(t)\|_{X_{p}}: 0 \leq t \leq T\right\}$.

\subsection{Full discretization}

In this section, we deduce stability estimates and bounds for the defect that are needed for the estimation of the contribution

$$
u_{M}^{n}-u^{n}=\left(\mathcal{Q}_{M}-I\right) u^{n}+\sum_{j=0}^{n-1} \prod_{\ell=j+1}^{n-1} \mathcal{F}_{M}\left(\tau_{\ell}\right)\left(\mathcal{F}_{M}\left(\tau_{j}\right) u^{j}-\mathcal{Q}_{M} \mathcal{S}\left(\tau_{j}\right) u^{j}\right)
$$

in the global error, associated with an additional spatial discretisation error, see also (6).

\subsubsection{Stability}

Estimates for the evolution operator associated with B. A first stability result for the composition of the spectral interpolation operator and the evolution operator associated with $B$ is provided by the following result. We recall the abbreviation $X_{M}=\operatorname{span}\left\{\mathcal{L}_{k m}^{\gamma}:(k, m) \in \mathcal{M}_{M}\right\}$. 
Lemma 13 For all $u, v \in X_{\alpha}$ with $\alpha \in \mathbb{N}_{\geq 1}$ the estimate

$$
\left\|\mathcal{Q}_{M}\left(\mathrm{e}^{-\mathrm{i} t B[u]} u-\mathrm{e}^{-\mathrm{i} t B[v]} v\right)\right\|_{X_{0}} \leq \mathrm{e}^{\mathcal{C}\left(\mathcal{C}_{V}+\mathcal{C}_{\alpha}^{2}|\beta|\right) t}\|u-v\|_{K+\frac{M}{2}, M}
$$

is valid with constants $\mathcal{C}_{V}>0$ and $\mathcal{C}_{\alpha}>0$ depending on upper bounds for $\|V\|_{X_{\alpha}}$ and $\|u\|_{X_{\alpha}}$ as well as $\|v\|_{X_{\alpha}}$. In particular, if $u, v \in X_{M}$, that $i s, \mathcal{P}_{M} u=\mathcal{Q}_{M} u=u$ and $\mathcal{P}_{M} v=\mathcal{Q}_{M} v=v$, the relation

$$
\left\|\mathcal{Q}_{M}\left(\mathrm{e}^{-\mathrm{i} t B[u]} u-\mathrm{e}^{-\mathrm{i} t B[v]} v\right)\right\|_{X_{0}} \leq \mathrm{e}^{\mathcal{C}\left(\mathcal{C}_{V}+\mathcal{C}_{\alpha}^{2}|\beta|\right) t}\|u-v\|_{X_{0}}
$$

follows.

Proof For simplicity, we assume $V=0$ and refer to [23] for the case $V \neq 0$. Let $\widetilde{u}(t)=\mathrm{e}^{-\mathrm{i} t B[u]} u$ and $\widetilde{v}(t)=\mathrm{e}^{-\mathrm{i} t B[v]} v$. By means of the variation-of-constants formula we obtain

$$
\begin{aligned}
& \left\|\mathcal{Q}_{M}(\widetilde{u}(t)-\widetilde{v}(t))\right\|_{X_{0}} \\
& \quad \leq\left\|\mathcal{Q}_{M} \mathrm{e}^{-\mathrm{i} t B[u]}(u-v)\right\|_{X_{0}} \\
& \quad \quad\|\| \mathcal{Q}_{M} \int_{0}^{t} \mathrm{e}^{-\mathrm{i}(t-\tau) B[u]}(B[u]-B[v]) \mathrm{e}^{-\mathrm{i} \tau B[v]} v \mathrm{~d} \tau \|_{X_{0}} .
\end{aligned}
$$

An application of Lemma 7 yields an estimate for the first term

$$
\begin{aligned}
\left\|\mathcal{Q}_{M} \mathrm{e}^{-\mathrm{i} t B[u]}(u-v)\right\|_{X_{0}}^{2} \leq\left\|\mathrm{e}^{-\mathrm{i} t B[u]}(u-v)\right\|_{K+\frac{M}{2}, M}^{2} \\
\quad=\frac{1}{M} \sum_{(j, s) \in \mathcal{K}_{K M}} w_{j}\left|\mathrm{e}^{-\mathrm{i} t B[u]\left(x_{j s}, y_{j s}\right)}\right|^{2}\left|(u-v)\left(x_{j s}, y_{j s}\right)\right|^{2} \\
\quad=\frac{1}{M} \sum_{(j, s) \in \mathcal{K}_{K M}} w_{j}\left|(u-v)\left(x_{j s}, y_{j s}\right)\right|^{2}=\|u-v\|_{K+\frac{M}{2}, M}^{2},
\end{aligned}
$$

see also (17) for the definition of the discrete $L^{2}$-norm. In a similar manner, together with Lemma 3 the bound

$$
\begin{aligned}
\| \mathcal{Q}_{M} & \int_{0}^{t} \mathrm{e}^{-\mathrm{i}(t-\tau) B[u]}(B[u]-B[v]) \mathrm{e}^{-\mathrm{i} \tau B[v]} v \mathrm{~d} \tau \|_{X_{0}} \\
& \leq\left\|\int_{0}^{t} \mathrm{e}^{-\mathrm{i}(t-\tau) B[u]}(B[u]-B[v]) \mathrm{e}^{-\mathrm{i} \tau B[v]} v \mathrm{~d} \tau\right\|_{K+\frac{M}{2}, M} \\
& \leq \int_{0}^{t}\left\|\mathrm{e}^{-\mathrm{i}(t-\tau) B[u]}(B[u]-B[v]) \mathrm{e}^{-\mathrm{i} \tau B[v]} v\right\|_{K+\frac{M}{2}, M} \mathrm{~d} \tau \\
& =\int_{0}^{t}\|(B[u]-B[v]) v\|_{K+\frac{M}{2}, M} \mathrm{~d} \tau \\
& =|\beta| t\|((u-v) \bar{u}+(\overline{u-v}) v) v\|_{K+\frac{M}{2}, M} \\
& \leq|\beta| t\left(\|u\|_{L^{\infty}\left(\mathcal{K}_{K M}\right)}+\|v\|_{L^{\infty}\left(\mathcal{K}_{K M}\right)}\right)\|v\|_{L^{\infty}\left(\mathcal{K}_{K M}\right)}\|u-v\|_{K+\frac{M}{2}, M} \\
& \leq \mathcal{C}|\beta| t\left(\|u\|_{X_{\alpha}}+\|v\|_{X_{\alpha}}\right)\|v\|_{X_{\alpha}}\|u-v\|_{K+\frac{M}{2}, M} \\
& \leq \mathcal{C} \mathcal{C}_{\alpha}^{2}|\beta| t\|u-v\|_{K+\frac{M}{2}, M}
\end{aligned}
$$

follows. Using that $1+x \leq \mathrm{e}^{x}$ this proves the first assertion, and the second statement then results by means of Lemma 7 . 
Stability of the discrete evolution operator. A fully discrete analogue to Lemma 10 is provided by the following auxiliary result; making use of the fact that the evolution operator associated with $A$ is unitary on $X_{0}$ and satisfies $(t \in \mathbb{R})$

$$
\mathrm{e}^{-\mathrm{i} t A} u \in X_{M}, \quad u \in X_{M},
$$

the statement follows immediately from Lemma 13.

Lemma 14 For all $u, v \in X_{M}$, that is, $\mathcal{P}_{M} u=\mathcal{Q}_{M} u=u$ as well as $\mathcal{P}_{M} v=$ $\mathcal{Q}_{M} v=v$, the estimate

$$
\left\|\mathcal{F}_{M}(t) u-\mathcal{F}_{M}(t) v\right\|_{X_{0}} \leq \mathrm{e}^{\mathcal{C}\left(\mathcal{C}_{V}+\mathcal{C}_{\alpha}^{2}|\beta|\right) t}\|u-v\|_{X_{0}}
$$

is valid with constants $\mathcal{C}_{V}>0$ and $\mathcal{C}_{\alpha}>0$ depending on upper bounds for $\|V\|_{X_{\alpha}}$ and $\|u\|_{X_{\alpha}}$ as well as $\|v\|_{X_{\alpha}}$, where $\alpha \in \mathbb{N}_{\geq 1}$.

\subsubsection{Local error}

Estimate for the defect. The following result provides a bound for the difference $\mathcal{F}_{M}(\Delta t) u-\mathcal{Q}_{M} \mathcal{S}(\Delta t) u$; auxiliary estimates are deduced below. We recall that the positive integers $(K, M) \in \mathbb{N}_{\geq 1} \times \mathbb{N}_{\geq 1}$ are required to be proportional.

Lemma 15 For any $u \in X_{\alpha}$ with $\alpha \in \mathbb{N}_{\geq 1}$ the estimate

$$
\begin{aligned}
& \left\|\mathcal{F}_{M}(\Delta t) u-\mathcal{Q}_{M} \mathcal{S}(\Delta t) u\right\|_{X_{0}} \\
& \quad \leq \mathcal{C} \mathcal{C}_{\alpha}\left(\mathcal{C}_{V}+\mathcal{C}_{\alpha}^{2}|\beta|\right) \mathrm{e}^{\mathcal{C}\left(\mathcal{C}_{V}+\mathcal{C}_{\alpha}^{2}|\beta|\right) \Delta t} \Delta t M^{-\left(\alpha-\frac{3}{2}\right)}
\end{aligned}
$$

is valid, where the constants $\mathcal{C}_{V}>0$ and $\mathcal{C}_{\alpha}>0$ depend on upper bounds for $\|V\|_{X_{\alpha}}$ and $\|u\|_{X_{\alpha}},\left\|\mathcal{Q}_{M} u\right\|_{X_{\alpha}}$, as well as $\left\|\mathcal{Q}_{M} \mathrm{e}^{-\mathrm{i} \frac{1}{2} \Delta t A} u\right\|_{X_{\alpha}}$, respectively.

Proof Employing the abbreviations

$$
z_{1}=\mathrm{e}^{-\mathrm{i} \Delta t B\left[z_{2}\right]} z_{2}, \quad z_{2}=\mathrm{e}^{-\mathrm{i} \frac{1}{2} \Delta t A} z_{3}, \quad z_{3}=u,
$$

as well as

$$
\begin{aligned}
Z_{1}= & \mathcal{Q}_{M} \mathrm{e}^{-\mathrm{i} \frac{1}{2} \Delta t A}\left(I-\mathcal{Q}_{M}\right) z_{1} \\
Z_{2}= & \mathcal{Q}_{M} \mathrm{e}^{-\mathrm{i} \frac{1}{2} \Delta t A} \mathcal{Q}_{M}\left(\mathrm{e}^{-\mathrm{i} \Delta t B\left[z_{2}\right]} z_{2}-\mathrm{e}^{-\mathrm{i} \Delta t B\left[\mathcal{Q}_{M} z_{2}\right]} \mathcal{Q}_{M} z_{2}\right), \\
Z_{3}= & \mathcal{Q}_{M} \mathrm{e}^{-\mathrm{i} \frac{1}{2} \Delta t A} \mathcal{Q}_{M}\left(\mathrm{e}^{-\mathrm{i} \Delta t B\left[\mathcal{Q}_{M} \mathrm{e}^{-\mathrm{i} \frac{1}{2} \Delta t A} z_{3}\right]} \mathcal{Q}_{M} \mathrm{e}^{-\mathrm{i} \frac{1}{2} \Delta t A} z_{3}\right. \\
& \left.\quad-\mathrm{e}^{-\mathrm{i} \Delta t B\left[\mathrm{e}^{-\mathrm{i} \frac{1}{2} \Delta t A} \mathcal{Q}_{M} z_{3}\right]} \mathrm{e}^{-\mathrm{i} \frac{1}{2} \Delta t A} \mathcal{Q}_{M} z_{3}\right),
\end{aligned}
$$

we have

$$
\mathcal{F}_{M}(\Delta t) u-\mathcal{Q}_{M} \mathcal{S}(\Delta t) u=-\left(Z_{1}+Z_{2}+Z_{3}\right) .
$$

Making use of the fact that $\mathrm{e}^{-\mathrm{i} \frac{1}{2} \Delta t A}$ preserves the norm on fractional power spaces and applying Lemma 16 as well as Lemma 9, we obtain

$$
\left\|Z_{1}\right\|_{X_{0}} \leq \mathcal{C} \Delta t M^{-\left(\alpha-\frac{3}{2}\right)}\left\|z_{1}\right\|_{X_{\alpha}} \leq \mathcal{C} \mathcal{C}_{\alpha} \mathrm{e}^{\mathcal{C}\left(\mathcal{C}_{V}+|\beta| \mathcal{C}_{\alpha}^{2}\right) \Delta t} \Delta t M^{-\left(\alpha-\frac{3}{2}\right)} .
$$


Using that $\left\|z_{2}\right\|_{X_{\alpha}}=\|u\|_{X_{\alpha}} \leq \mathcal{C}_{\alpha}$ and $\left\|\mathcal{Q}_{M} \mathrm{e}^{-\mathrm{i} \frac{1}{2} \Delta t A} \mathcal{Q}_{M} v\right\|_{X_{0}}=\left\|\mathcal{Q}_{M} v\right\|_{X_{0}}$, an application of Lemma 18 yields

$$
\left\|Z_{2}\right\|_{X_{0}} \leq \mathcal{C} \mathcal{C}_{\alpha}\left(\mathcal{C}_{V}+|\beta| \mathcal{C}_{\alpha}^{2}\right) \mathrm{e}^{\mathcal{C}\left(\mathcal{C}_{V}+|\beta| \mathcal{C}_{\alpha}^{2}\right) \Delta t} \Delta t M^{-\left(\alpha-\frac{3}{2}\right)} .
$$

Together with Lemma 13 and Lemma 16 the bound

$$
\begin{aligned}
\left\|Z_{3}\right\|_{X_{0}} & \leq \mathrm{e}^{\mathcal{C C}_{a}^{2}|\beta| \Delta t}\left\|\mathcal{Q}_{M} \mathrm{e}^{-\mathrm{i} \frac{1}{2} \Delta t A}\left(\mathcal{Q}_{M}-I\right) u\right\|_{X_{0}} \\
& \leq \mathcal{C} \mathcal{C}_{\alpha} \mathrm{e}^{\mathcal{C} \Delta t|\beta| \mathcal{C}_{a}^{2}} \Delta t M^{-\left(\alpha-\frac{3}{2}\right)}
\end{aligned}
$$

follows; we note that the constant $\mathcal{C}_{\alpha}$ also provides a bound for $\left\|\mathcal{Q}_{M} u\right\|_{X_{\alpha}}$ and $\left\|\mathcal{Q}_{M} \mathrm{e}^{-\mathrm{i} \frac{1}{2} \Delta t A} u\right\|_{X_{\alpha}}$. Altogether, this proves the assertion.

Auxiliary estimates for the proof of Lemma 15 are provided by the following results.

Lemma 16 For any $u \in X_{\alpha}$ with $\alpha \in \mathbb{N}_{\geq 1}$ the relation

$$
\left\|\mathcal{Q}_{M} \mathrm{e}^{-\mathrm{i} t A}\left(\mathcal{Q}_{M}-I\right) u\right\|_{X_{0}} \leq \mathcal{C} t M^{-\left(\alpha-\frac{3}{2}\right)}\|u\|_{X_{\alpha}}
$$

holds.

Proof We utilize that the function which corresponds to the considered composition

$$
\begin{gathered}
\eta(t)=\mathcal{Q}_{M} \mathrm{e}^{-\mathrm{i} t A}\left(\mathcal{Q}_{M}-I\right) u=v(t)-\mathcal{Q}_{M} w(t), \\
v(t)=\mathcal{Q}_{M} \mathrm{e}^{-\mathrm{i} t A} \mathcal{Q}_{M} u=\mathrm{e}^{-\mathrm{i} t A} \mathcal{Q}_{M} u, \quad w(t)=\mathrm{e}^{-\mathrm{i} t A} u,
\end{gathered}
$$

is a solution to the initial value problem

$$
\mathrm{i} \frac{\mathrm{d}}{\mathrm{d} t} \eta(t)=A \eta(t)+\left[A, \mathcal{Q}_{M}\right] w(t), \quad \eta(0)=v(0)-\mathcal{Q}_{M} w(0)=0,
$$

since $\mathrm{i} \frac{\mathrm{d}}{\mathrm{d} t} v(t)=A v(t)$ and

$$
\mathrm{i} \frac{\mathrm{d}}{\mathrm{d} t}\left(\mathcal{Q}_{M} w(t)\right)=\mathcal{Q}_{M} A w(t)=A \mathcal{Q}_{M} w(t)-\left[A, \mathcal{Q}_{M}\right] w(t) .
$$

The variation-of-constants formula thus implies

$$
\eta(t)=\int_{0}^{t} \mathrm{e}^{-\mathrm{i}(t-\tau) A}\left[A, \mathcal{Q}_{M}\right] w(\tau) \mathrm{d} \tau=\int_{0}^{t} \mathrm{e}^{-\mathrm{i}(t-\tau) A}\left[A, \mathcal{Q}_{M}\right] \mathrm{e}^{-\mathrm{i} \tau A} u \mathrm{~d} \tau .
$$

From Lemma 8 we conclude that the bound

$$
\begin{aligned}
\left\|\left[A, \mathcal{Q}_{M}\right] u\right\|_{X_{0}} & =\left\|A \mathcal{Q}_{M} u-A u+A u-\mathcal{Q}_{M} A u\right\|_{X_{0}} \\
& \leq\left\|A\left(\mathcal{Q}_{M}-I\right) u\right\|_{X_{0}}+\left\|\left(\mathcal{Q}_{M}-I\right) A u\right\|_{X_{0}} \\
& \leq \mathcal{C} M^{-\left(\alpha-\frac{3}{2}\right)}\|u\|_{X_{\alpha}}
\end{aligned}
$$

holds, which further yields

$$
\|\eta(t)\|_{X_{0}} \leq \int_{0}^{t}\left\|\left[A, \mathcal{Q}_{M}\right] \mathrm{e}^{-\mathrm{i} \tau A} u\right\|_{X_{0}} \mathrm{~d} \tau \leq \mathcal{C} t M^{-\left(\alpha-\frac{3}{2}\right)}\|u\|_{X_{\alpha}}
$$

and thus proves the stated result. 
Lemma 17 For any $u \in X_{\alpha}$ with $\alpha \in \mathbb{N}_{\geq 1}$ the estimate

$\left\|\left(\mathcal{Q}_{M}-I\right) \mathrm{e}^{-\mathrm{i} t B\left[\mathcal{Q}_{M} u\right]} \mathcal{Q}_{M} u\right\|_{X_{0}} \leq \mathcal{C} \mathcal{C}_{\alpha}\left(\mathcal{C}_{V}+\mathcal{C}_{\alpha}^{2}|\beta|\right) \mathrm{e}^{\mathcal{C}\left(\mathcal{C}_{V}+\mathcal{C}_{\alpha}^{2}|\beta|\right) t} t M^{-\left(\alpha-\frac{1}{2}\right)}$

holds, where the constants $\mathcal{C}_{V}>$ and $\mathcal{C}_{\alpha}>0$ depend on upper bounds for $\|V\|_{X_{\alpha}}$ and $\|u\|_{X_{\alpha}}$ as well as $\left\|\mathcal{Q}_{M} u\right\|_{X_{\alpha}}$, respectively.

Proof We set $v=\mathcal{Q}_{M} u$ and utilize that $\eta(t)=\left(\mathrm{e}^{-\mathrm{i} t B[v]}-I\right) v$ is the solution to the initial value problem

$$
\mathrm{i} \frac{\mathrm{d}}{\mathrm{d} t} \eta(t)=B[v] \eta(t)+B[v] v, \quad \eta(0)=0,
$$

with the following representation by the variation-of-constants formula

$$
\eta(t)=\int_{0}^{t} \mathrm{e}^{-\mathrm{i}(t-\tau) B[v]} B[v] v \mathrm{~d} \tau=\int_{0}^{t} B[v] \mathrm{e}^{-\mathrm{i}(t-\tau) B[v]} v \mathrm{~d} \tau .
$$

By Lemma 8 we obtain the estimate

$$
\begin{aligned}
& \left\|\left(\mathcal{Q}_{M}-I\right) \mathrm{e}^{-\mathrm{i} t B[u]} v\right\|_{X_{0}}=\left\|\left(\mathcal{Q}_{M}-I\right)\left(\mathrm{e}^{-\mathrm{i} t B[v]}-I\right) v\right\|_{X_{0}} \\
& \quad \leq \mathcal{C} M^{-\left(\alpha-\frac{1}{2}\right)}\left\|\left(\mathrm{e}^{-\mathrm{i} t B[v]}-I\right) v\right\|_{X_{\alpha}}=\mathcal{C} M^{-\left(\alpha-\frac{1}{2}\right)}\|\eta(t)\|_{X_{\alpha}}
\end{aligned}
$$

together with Lemma 9 this yields

$$
\begin{aligned}
\|\eta(t)\|_{X_{\alpha}} & \leq \mathcal{C}\left(\mathcal{C}_{V}+\mathcal{C}_{\alpha}^{2}|\beta|\right) \int_{0}^{t} \mathrm{e}^{\mathcal{C}\left(\mathcal{C}_{V}+\mathcal{C}_{\alpha}^{2}|\beta|\right) \tau}\|v\|_{X_{\alpha}} \mathrm{d} \tau \\
& \leq \mathcal{C} \mathcal{C}_{\alpha}\left(\mathcal{C}_{V}+\mathcal{C}_{\alpha}^{2}|\beta|\right) \mathrm{e}^{\mathcal{C}\left(\mathcal{C}_{V}+\mathcal{C}_{\alpha}^{2}|\beta|\right) t} t
\end{aligned}
$$

which proves the stated result.

Lemma 18 For any $u \in X_{\alpha}$ with $\alpha \in \mathbb{N}_{\geq 1}$ the estimate

$$
\begin{aligned}
& \left\|\mathcal{Q}_{M}\left(\mathrm{e}^{-\mathrm{i} t B[u]} u-\mathrm{e}^{-\mathrm{i} t B\left[\mathcal{Q}_{M} u\right]} \mathcal{Q}_{M} u\right)\right\|_{X_{0}} \\
& \quad \leq \mathcal{C} \mathcal{C}_{\alpha}\left(\mathcal{C}_{V}+\mathcal{C}_{\alpha}^{2}|\beta|\right) \mathrm{e}^{\mathcal{C}\left(\mathcal{C}_{V}+\mathcal{C}_{\alpha}^{2}|\beta|\right) t} t M^{-\left(\alpha-\frac{3}{2}\right)}
\end{aligned}
$$

is valid, where the constants $\mathcal{C}_{V}>$ and $\mathcal{C}_{\alpha}>0$ depend on upper bounds for $\|V\|_{X_{\alpha}}$ and $\|u\|_{X_{\alpha}}$ as well as $\left\|\mathcal{Q}_{M} u\right\|_{X_{\alpha}}$, respectively.

Proof Similarly as before, we introduce the auxiliary functions

$$
v(t)=\mathrm{e}^{-\mathrm{i} t B[u]} u, \quad w(t)=\mathrm{e}^{-\mathrm{i} t B\left[\mathcal{Q}_{M} u\right]} \mathcal{Q}_{M} u, \quad \eta(t)=\mathcal{Q}_{M}(v(t)-w(t)),
$$

and use that $\eta$ is a solution to the evolution equation

$$
\begin{aligned}
\mathrm{i} \frac{\mathrm{d}}{\mathrm{d} t} \eta(t) & =B[u] \eta(t)-\left[B[u], \mathcal{Q}_{M}\right] v(t)+\left(B[u] \mathcal{Q}_{M}-\mathcal{Q}_{M} B\left[\mathcal{Q}_{M} u\right]\right) w(t) \\
& =B[u] \eta(t)-\left[B[u], \mathcal{Q}_{M}\right](v(t)-w(t))+\mathcal{Q}_{M}\left(B[u]-B\left[\mathcal{Q}_{M} u\right]\right) w(t)
\end{aligned}
$$


with initial value $\eta(0)=0$. As a consequence, an application of the variationof-constants formula yields the integral representation

$$
\begin{aligned}
\eta(t)= & \int_{0}^{t} \mathrm{e}^{-\mathrm{i}(t-\tau) B[u]} \\
& \times\left(-\left[B[u], \mathcal{Q}_{M}\right](v(\tau)-w(\tau))+\mathcal{Q}_{M}\left(B[u]-B\left[\mathcal{Q}_{M} u\right]\right) w(\tau)\right) \mathrm{d} \tau .
\end{aligned}
$$

Making use of the fact that $\eta(t)=\mathcal{Q}_{M} \eta(t)$ and that the operator $\mathrm{e}^{-\mathrm{i}(t-\tau) B[u]}$ is unitary with respect to the discrete $L^{2}$-norm, the bound provided by Lemma 7 yields

$$
\begin{aligned}
\|\eta(t)\|_{X_{0}} \leq \int_{0}^{t} & \left\|\left[B[u], \mathcal{Q}_{M}\right](v(\tau)-w(\tau))\right\|_{K+\frac{M}{2}, M} \mathrm{~d} \tau \\
& +\int_{0}^{t}\left\|\left(B[u]-B\left[\mathcal{Q}_{M} u\right]\right) w(\tau)\right\|_{K+\frac{M}{2}, M} \mathrm{~d} \tau .
\end{aligned}
$$

Using that by Lemma 8 the relation

$$
\left\|\left(\mathcal{Q}_{M}-I\right) u\right\|_{K+\frac{M}{2}, M} \leq \mathcal{C}\left\|\left(\mathcal{Q}_{M}-I\right) u\right\|_{X_{1}} \leq \mathcal{C} M^{-\left(\alpha-\frac{3}{2}\right)}\|u\|_{X_{\alpha}}
$$

holds, arguments close to the proof of Lemma 13 lead to the following bound for the second integral

$$
\begin{aligned}
\int_{0}^{t} \| & \left(B[u]-B\left[\mathcal{Q}_{M} u\right]\right) \mathrm{e}^{-\mathrm{i} \tau B\left[\mathcal{Q}_{M} u\right]} \mathcal{Q}_{M} u \|_{K+\frac{M}{2}, M} \mathrm{~d} \tau \\
& \leq \mathcal{C} \mathcal{C}_{\alpha}^{2}|\beta| t\left\|\left(\mathcal{Q}_{M}-I\right) u\right\|_{K+\frac{M}{2}, M} \\
& \leq \mathcal{C} \mathcal{C}_{\alpha}^{3}|\beta| t M^{-\left(\alpha-\frac{3}{2}\right)} \\
& \leq \mathcal{C} \mathcal{C}_{\alpha}\left(\mathcal{C}_{V}+|\beta| \mathcal{C}_{\alpha}^{2}\right) \mathrm{e}^{\mathcal{C}\left(\mathcal{C}_{V}+|\beta| \mathcal{C}_{\alpha}^{2}\right) t} t M^{-\left(\alpha-\frac{3}{2}\right)} .
\end{aligned}
$$

In order to estimate the first integral we employ the bound

$$
\begin{aligned}
& \left\|\left[B[u], \mathcal{Q}_{M}\right] v\right\|_{K+\frac{M}{2}, M} \\
& \quad \leq\left\|B[u]\left(\mathcal{Q}_{M}-I\right) v\right\|_{K+\frac{M}{2}, M}+\left\|\left(\mathcal{Q}_{M}-I\right) B[u] v\right\|_{K+\frac{M}{2}, M} \\
& \quad \leq \mathcal{C}\left(\|V\|_{X_{\alpha}}+|\beta|\|u\|_{X_{\alpha}}^{2}\right)\left\|\left(\mathcal{Q}_{M}-I\right) v\right\|_{K+\frac{M}{2}, M}+\mathcal{C} M^{-\left(\alpha-\frac{3}{2}\right)}\|B[u] v\|_{X_{\alpha}} \\
& \quad \leq \mathcal{C} M^{-\left(\alpha-\frac{3}{2}\right)}\left(\|V\|_{X_{\alpha}}+|\beta|\|u\|_{X_{\alpha}}^{2}\right)\|v\|_{X_{\alpha}} \\
& \quad \leq \mathcal{C} M^{-\left(\alpha-\frac{3}{2}\right)}\left(\mathcal{C}_{V}+\mathcal{C}_{\alpha}^{2}|\beta|\right)\|v\|_{X_{\alpha}},
\end{aligned}
$$

see also Lemma 8 and Lemma 9, and again by Lemma 9 obtain

$$
\begin{aligned}
\|v(\tau)-w(\tau)\|_{X_{\alpha}} & \leq\left\|\mathrm{e}^{-\mathrm{i} \tau B[u]} u\right\|_{X_{\alpha}}+\left\|\mathrm{e}^{-\mathrm{i} \tau B\left[\mathcal{Q}_{M} u\right]} \mathcal{Q}_{M} u\right\|_{X_{\alpha}} \\
& \leq 2 \mathcal{C}_{\alpha} \mathrm{e}^{\mathcal{C}\left(\mathcal{C}_{V}+\mathcal{C}_{\alpha}^{2}|\beta|\right) \tau} ;
\end{aligned}
$$


this implies the estimate

$$
\begin{aligned}
\int_{0}^{t} \| & {\left[B[u], \mathcal{Q}_{M}\right](v(\tau)-w(\tau)) \|_{K+\frac{M}{2}, M} \mathrm{~d} \tau } \\
& \leq \mathcal{C} \mathcal{C}_{\alpha}\left(\mathcal{C}_{V}+|\beta| \mathcal{C}_{\alpha}^{2}\right) \mathrm{e}^{\mathcal{C}\left(\mathcal{C}_{V}+|\beta| \mathcal{C}_{\alpha}^{2}\right) t} t M^{-\left(\alpha-\frac{3}{2}\right)} .
\end{aligned}
$$

Altogether, this proves the assertion.

5.3 Extension to three space dimensions

In this section, we study the generalized-Laguerre-Fourier-Hermite pseudospectral method for the space discretization of the three-dimensional GrossPitaevskii equation with rotation term. As our error analysis for the twodimensional case naturally carries over to the case of three space dimensions, we only indicate where estimates have to be extended with some care. For additional details on the definition of the Hermite polynomials and the numerical computation of the corresponding quadrature nodes and weights we refer to [11]. We recall (1)-(2) and in particular formula (3), where the eigenfunctions and associated eigenvalues of the linear operator $A$ defined in (2b) are specified.

Basic relations. For the time-dependent three-dimensional Gross-Pitaevskii equation with rotation term the discretization of the $(x, y)$-variables relies on the generalized-Laguerre-Fourier spectral method analyzed before, and the discretization of the $z$-variable uses scaled Hermite functions involving the Hermite polynomial of degree $\ell \in \mathbb{N}$. Analogously to (19), assuming that the discretization parameters $(K, M, L) \in \mathbb{N}_{\geq 1} \times \mathbb{N}_{\geq 1} \times \mathbb{N}_{\geq 1}$ are proportional with $M$ even, we introduce the index sets

$$
\begin{gathered}
\mathcal{M}_{M}=\{0,1, \ldots, K-1\} \times\left\{-\frac{M}{2},-\frac{M}{2}+1, \ldots, \frac{M}{2}-1\right\} \times\{0,1, \ldots, L-1\}, \\
\mathcal{K}_{M}=\left\{0,1, \ldots, K+\frac{M}{2}-1\right\} \times\{0,1, \ldots, M-1\} \times\{0,1, \ldots, L-1\} .
\end{gathered}
$$

In the present situation, the maximum eigenvalue in the set $\mathcal{M}_{M}$ satisfies the relation

$$
\lambda_{\max }=\max _{(k, m, \ell) \in \mathcal{M}_{M}} \lambda_{k m \ell} \leq \mathcal{C}(K+M+L) \leq \mathcal{C} M,
$$

see also Lemma 8, and the spectral interpolant given by

$$
\begin{gathered}
\mathcal{Q}_{M} u=\sum_{(k, m, \ell) \in \mathcal{M}_{M}} \widetilde{c}_{k m}(u) \mathcal{B}_{k m \ell}^{\gamma, \gamma_{z}}, \\
\widetilde{c}_{k m \ell}(u)=\frac{1}{M} \sum_{(r, s, q) \in \mathcal{K}_{M}} w_{r} \widetilde{w}_{q} \overline{\mathcal{B}_{k m \ell}^{\gamma, \gamma}\left(x_{r s}, y_{r s}, z_{q}\right)} u\left(x_{r s}, y_{r s}, z_{q}\right),
\end{gathered}
$$

in addition involves scaled Gauß-Hermite quadrature weights and nodes $\left(\widetilde{w}_{q}, z_{q}\right)_{q=1}^{L}$, see also $(20)$. 
Error analysis. By means of recurrence relations for scaled Hermite functions, analogous to Lemma 1, arguments in the lines of the proof of Lemma 2 yield the estimate

$$
\|z u\|_{X_{\alpha}}+\left\|\partial_{z} u\right\|_{X_{\alpha}} \leq \mathcal{C}\|u\|_{X_{\alpha+\frac{1}{2}}}, \quad u \in X_{\alpha+\frac{1}{2}}
$$

and further imply Lemma 3, see also $[10,23]$. In order to extend the relations for the spectral interpolant stated in Lemma 7 and Lemma 8, we utilize the bound

$$
\sum_{q=0}^{L-1} \widetilde{w}_{q}\left|v\left(z_{q}\right)\right|^{2} \leq \mathcal{C} \int_{\mathbb{R}}\left(|v(z)|^{2}+M^{-\frac{1}{3}}\left|\partial_{z} v(z)\right|^{2}\right) \mathrm{d} z, \quad v \in H^{1}(\mathbb{R})
$$

deduced in [12], which implies the following estimate for the associated discrete $L^{2}$-norm

$$
\begin{aligned}
\|u\|_{M}^{2}= & \frac{1}{M} \sum_{(r, s, q) \in \mathcal{K}_{M}} w_{r} \widetilde{w}_{q}\left|u\left(x_{r s}, y_{r s}, z_{q}\right)\right|^{2} \\
\leq \mathcal{C} & \sum_{q=0}^{L-1} \widetilde{w}_{q} \int_{\mathbb{R}^{2}}\left(\left|u\left(\cdot, z_{q}\right)\right|^{2}+M^{-\frac{1}{3}}\left|\partial_{x} u\left(\cdot, z_{q}\right)\right|^{2}+M^{-\frac{1}{3}}\left|\partial_{y} u\left(\cdot, z_{q}\right)\right|^{2}\right. \\
& \left.\quad+M^{-1}\left|\partial_{x}^{2} u\left(\cdot, z_{q}\right)\right|^{2}+M^{-1}\left|\partial_{y}^{2} u\left(\cdot, z_{q}\right)\right|^{2}+M^{-1}\left|\partial_{x y} u\left(\cdot, z_{q}\right)\right|^{2}\right) \\
\leq \mathcal{C} & \int_{\mathbb{R}^{3}}\left(|u|^{2}+M^{-\frac{1}{3}}\left|\partial_{x} u\right|^{2}+M^{-\frac{1}{3}}\left|\partial_{y} u\right|^{2}+M^{-\frac{1}{3}}\left|\partial_{z} u\right|^{2}\right. \\
& \quad+M^{-1}\left|\partial_{x}^{2} u\right|^{2}+M^{-1}\left|\partial_{y}^{2} u\right|^{2}+M^{-1}\left|\partial_{x y} u\right|^{2} \\
& \quad+M^{-\frac{2}{3}}\left|\partial_{x z} u\right|^{2}+M^{-\frac{2}{3}}\left|\partial_{y z} u\right|^{2} \\
& \left.+M^{-\frac{4}{3}}\left|\partial_{x x z} u\right|^{2}+M^{-\frac{4}{3}}\left|\partial_{y y z} u\right|^{2}+M^{-\frac{4}{3}}\left|\partial_{x y z} u\right|^{2}\right) \\
\leq \mathcal{C} & \left(\|u\|_{L^{2}}^{2}+M^{-\frac{1}{3}}|u|_{H^{1}}^{2}+M^{-1}|u|_{H^{2}}^{2}+M^{-\frac{4}{3}}|u|_{H^{3}}^{2}\right)
\end{aligned}
$$

which further yields

$$
\begin{aligned}
\left\|\left(\mathcal{Q}_{M}-I\right) u\right\|_{X_{\zeta}} \leq \mathcal{C} \lambda_{\max }^{-(\alpha-\zeta)}\left(1+\lambda_{\max }^{\frac{1}{2}} M^{-\frac{1}{6}}\right. \\
\\
\left.\quad+\lambda_{\max } M^{-\frac{1}{2}}+\lambda_{\max }^{\frac{3}{2}} M^{-\frac{2}{3}}\right)\|u\|_{X_{\alpha}} \\
\leq \mathcal{C} M^{-\left(\alpha-\zeta-\frac{5}{6}\right)}\|u\|_{X_{\alpha}} .
\end{aligned}
$$

As a consequence, in the three-dimensional case the factors $M^{-\left(\alpha-\frac{1}{2}\right)}$ and $M^{-\left(\alpha-\frac{3}{2}\right)}$ arising in the auxiliary results derived in Section 5.2 have to be replaced by $M^{-\left(\alpha-\frac{5}{6}\right)}$ and $M^{-\left(\alpha-\frac{11}{6}\right)}$, respectively. Altogether, this proves the statement of Theorem 1 . 


\section{References}

1. M. Abramowitz and I.A. Stegun. Handbook of mathematical functions: with formulas, graphs, and mathematical tables. Dover Publications, N.Y., 1992. Reprint of the 1972 edition.

2. R.A. Adams. Sobolev Spaces. Academic Press, Orlando, Fla., 1975.

3. W. Bao and Y. Cai. Mathematical theory and numerical methods for Bose-Einstein condensation. Kinet. Relat. Mod., 6:1-135, 2013.

4. W. Bao, H. Li, and J. Shen. A generalized-Laguerre-Fourier-Hermite pseudospectral method for computing the dynamics of rotating Bose-Einstein condensates. SIAM J. Sci. Comput., 31(5):3685-3711, 2009.

5. W. Bao and J. Shen. A fourth-order time-splitting Laguerre-Hermite pseudospectral method for Bose-Einstein condensates. SIAM J. Sci. Comput., 26(6):2010-2028, 2005.

6. S. Blanes and P.C. Moan. Practical symplectic partitioned Runge-Kutta and RungeKutta-Nyström methods. J. Comput. Appl. Math., 142:313-330, 2002.

7. M. Caliari, Ch. Neuhauser, and M. Thalhammer. High-order time-splitting Hermite and Fourier spectral methods for the Gross-Pitaevskii equation. J. Comput. Phys., 228:822-832, 2009.

8. I. Danaila and F. Hecht. A finite element method with mesh adaptivity for computing vortex states in fast-rotating Bose-Einstein condensates. J. Comput. Phys., 229:6946$6960,2010$.

9. I. Danaila and P. Kazemi. A new Sobolev gradient method for direct minimization of the Gross-Pitaevskii energy with rotation. SIAM J. Sci. Comput., 32:2447-2467, 2010.

10. L. Gauckler. Convergence of a split-step Hermite method for the Gross-Pitaevskii equation. IMA J. Numer. Anal., 31:396-415, 2011.

11. W. Gautschi. Orthogonal polynomials : computation and approximation. Oxford University Press, Oxford, U.K., 2004

12. B. Guo, J. Shen, and Ch. Xu. Spectral and pseudospectral approximations using Hermite functions: Application to the Dirac equation. Advances in Comput. Math., 19:3555, 2003.

13. E. Hairer, C. Lubich, and G. Wanner. Geometric Numerical Integration. SpringerVerlag, Berlin-Heidelberg-New York, 2002.

14. O. Koch, Ch. Neuhauser, and M. Thalhammer. Error analysis of high-order splitting methods for nonlinear evolutionary Schrödinger equations and application to the MCTDHF equations in electron dynamics. To appear in M2AN Math. Model. Numer. Anal., 2012 .

15. E. Levin and D. Lubinsky. Orthogonal polynomials for exponential weights $x^{2 \rho} e^{-2 Q(x)}$ on $[0, d)$. J. Approx. Theory, 134(2):199-256, 2005.

16. E. Levin and D. Lubinsky. Orthogonal polynomials for exponential weights $x^{2 \rho} e^{-2 Q(x)}$ on $[0, d)$, II. J. Approx. Theory, 139(1-2):107-143, 2006.

17. C. Lubich. On splitting methods for Schrödinger-Poisson and cubic nonlinear Schrödinger equations. Math. Comp., 77:2141-2153, 2008.

18. R. McLachlan and R. Quispel. Splitting methods. Acta Numer., 11:341-434, 2002.

19. F.W.J. Olver, D.W. Lozier, R.F. Boisvert, and C.W. Clark. NIST Handbook of Mathematical Functions. Cambridge University Press, Cambridge, N.Y., 2010.

20. R. Remmert. Theory of Complex Functions. Springer Verlag, New York, 1991.

21. J. Shen, T. Tang, and L. Wang. Spectral Methods: Algorithms, Analysis and Applications. Springer Verlag, Berlin, 2011.

22. M. Thalhammer. High-order exponential operator splitting methods for time-dependent Schrödinger equations. SIAM J. Numer. Anal., 46(4):2022-2038, 2008.

23. M. Thalhammer. Convergence analysis of high-order time-splitting pseudo-spectral methods for nonlinear Schrödinger equations. SIAM J. Numer. Anal., 50:3231-3258, 2012 .

24. H. Triebel. Higher Analysis. Barth, Leipzig-Berlin-Heidelberg, 1992. 